\title{
HAMILTONIAN CYCLES AND SUBSETS OF DISCOUNTED OCCUPATIONAL MEASURES
}

\author{
ALI ESHRAGH ${ }^{1}$, JERZY A. FILAR ${ }^{2}$, THOMAS KALINOWSKI ${ }^{1,3}$, AND SOGOL MOHAMMADIAN ${ }^{1}$
}

\begin{abstract}
We study a certain polytope arising from embedding the Hamiltonian cycle problem in a discounted Markov decision process. The Hamiltonian cycle problem can be reduced to finding particular extreme points of a certain polytope associated with the input graph. This polytope is a subset of the space of discounted occupational measures. We characterize the feasible bases of the polytope for a general input graph $G$, and determine the expected numbers of different types of feasible bases when the underlying graph is random. We utilize these results to demonstrate that augmenting certain additional constraints to reduce the polyhedral domain can eliminate a large number of feasible bases that do not correspond to Hamiltonian cycles. Finally, we develop a random walk algorithm on the feasible bases of the reduced polytope and present some numerical results. We conclude with a conjecture on the feasible bases of the reduced polytope.
\end{abstract}

\section{INTRODUCTION.}

One of the classical problems of combinatorial mathematics is the Hamiltonian Cycle Problem (HCP), named after the Irish mathematician, Sir William Rowan Hamilton. He designed the Icosian Game. To win this game, a player must visit each of twenty specifically connected cities, represented by holes on a wooden pegboard, exactly once and return to the starting point. The Hamiltonian cycle problem is a mathematically generalized version of this game. Given a graph $G$, the aim is either to find a cycle that passes through every node of $G$ exactly once, or to determine that no such cycle exists. Cycles that pass through every node of the graph exactly once are called Hamiltonian cycles. If the graph contains at least one Hamiltonian cycle, then it is called Hamiltonian. Otherwise, it is non-Hamiltonian. Figures 1(a) and 1(b) show examples of Hamiltonian and non-Hamiltonian graphs on five nodes.

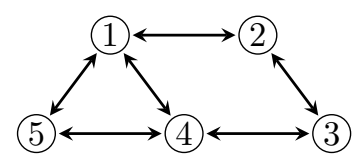

(a) A Hamiltonian graph

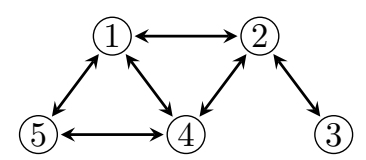

(b) A non-Hamiltonian graph

FiguRE 1. Hamiltonian and non-Hamiltonian graphs

Despite originating in the $1850 \mathrm{~s}, \mathrm{HCP}$ continues to generate a great deal of research interest. The similarity between HCP and the famous Traveling Salesman Problem (TSP) makes it interesting from a combinatorial optimization viewpoint. As TSP aims to find a route of minimal distance for a salesman who starts from a home location, visits every city exactly once and returns to the home location, HCP can be considered a special case of this problem. To see this, for a given graph $G$, add artificial $\operatorname{arcs}(i, j)$ for every pair $(i, j)$ of distinct nodes which are not connected by an arc in $G$. If we assign distance one to each original arc and

\footnotetext{
${ }^{1}$ School of Mathematical and Physical Sciences, University of Newcastle, NSW, Australia

${ }^{2}$ School of Mathematics and Physics, University of Queensland, QLD, Australia

${ }^{3}$ School of Science and Technology, University of New England, NSW, Australia

E-mail addresses: \{ali.eshragh,sogol.mohammadian\}@newcastle.edu.au, j.filar@uq.edu.au, t.kalinow@une.edu.au. Date: January 29, 2019.

2010 Mathematics Subject Classification. 90C40, 90C35; secondary: 05C80, 05C81.

Key words and phrases. Hamiltonian cycle, discounted occupational measures, feasible basis, random graph, random walk.

The second author wishes to acknowledge the support from the Australian Research Council under grants DP150100618 and DP160101236.
} 
distance two to each artificial arc, the graph $G$ is Hamiltonian if and only if there is a shortest route in the modified graph with the total distance of $n$.

In 1976, Garey et al. [17] showed that HCP is an NP-complete problem. In particular, no-one has found a solution algorithm with polynomial worst-case runtime. Its simple appearance, however, has captivated many researchers, giving rise to a rich literature presenting theoretical and computational results about this problem. In particular, TSP has been one of the major driving forces for the development of polyhedral theory for combinatorial optimization. The TSP polytope is the convex hull of the Hamiltonian cycles of a complete graph, and the great success with solving large instances of this problem is based on the deep insights into the geometry and combinatorics of this polytope (see [3]).

Arguably, one of the central problems in theoretical Operations Research is TSP. While TSP has received a lot of attention in the literature, much of its difficulty lies in the 'visit all nodes exactly once' constraint that is captured mathematically in HCP. Since HCP is known to be NP-complete, probabilistic embedding approaches such as the line of research continued in the present paper offers a pathway towards deeper understanding of the essential difficulty of both HCP and TSP.

In 1994, Filar and Krass [15] proposed a new approach to HCP, embedding it into a Markov Decision Process (MDP). An MDP comprises a state space, an action space, a function of transition probabilities between states (conditioned on the actions taken by the decision maker) and a reward function. In the MDP's basic setting, the decision maker takes an action, receives a reward from the environment, and the environment changes its state. Next, the decision maker identifies the state of the environment, takes a further action, obtains a reward, and so forth. The state transitions are probabilistic, and depend solely on the actual state and the action taken by the decision maker. The reward obtained by the decision maker depends on the action taken, and on the current state of the environment. The decision maker's actions in each environmental state are prescribed by a policy. Markov decision processes are applicable to a wide range of optimization problems. The model introduced in Filar and Krass [15] instigated a new line of research, which has attracted growing attention (see, for example, $[4,7,8,10,9,11,14,16,20]$ ).

In 2000, Feinberg [14] investigated the relationship between HCP and discounted MDPs. In discounted MDPs, a discount factor $\beta \in(0,1)$, which represents the difference in importance between future and present rewards, is used to discount rewards. Feinberg showed that HCP can be viewed as a discounted MDP with constraints. Part of his proof involved the construction of a new polytope corresponding to a given graph $G$, which we shall refer to as $\mathcal{F}_{\beta}(G)$. The polytope $\mathcal{F}_{\beta}(G)$ is a subset of the space of discounted occupational measures induced by the constrained discounted MDP. Feinberg showed that if the graph $G$ is Hamiltonian, the polytope $\mathcal{F}_{\beta}(G)$ has an extreme point, called a Hamiltonian extreme point, for each of its Hamiltonian cycles. Subsequently, Ejov et al. [10] described some geometric properties of $\mathcal{F}_{\beta}(G)$ and Eshragh et al. [13] transformed $\mathcal{F}_{\beta}(G)$ to a polytope $\mathcal{H}_{\beta}(G)$ to improve algorithmic efficiency. In 2011, Eshragh and Filar [12] partitioned all extreme points of $\mathcal{H}_{\beta}(G)$ into five types, consisting of Hamiltonian extreme points and non-Hamiltonian extreme points of types $1,2,3$ and 4 . They constructed a new polytope $\mathcal{W H}_{\beta}(G)$ by adding new linear constraints, called wedge constraints, to the polytope $\mathcal{H}_{\beta}(G)$. They showed that, when the discount factor $\beta$ is sufficiently close to one, the wedge constraints remove the, typically, most abundant non-Hamiltonian extreme points of types 2,3 and 4 , while preserving the Hamiltonian extreme points.

In this paper, we develop geometric properties of the feasible bases of the polytope $\mathcal{H}_{\beta}(G)$ and characterize them. Moreover, we find the expected number of feasible bases associated with Hamiltonian extreme points as well as each type of non-Hamiltonian extreme point in the random polytope $\mathcal{H}_{\beta}\left(G_{n, p}\right)$ for an input binomial random graph $G_{n, p}$. We show that in expectation, the feasible bases corresponding to non-Hamiltonian extreme points of Type 4 are the majority. Motivated by the results of Eshragh and Filar [12], we construct two algorithms based on a simple random walk on feasible bases of the two polytopes $\mathcal{H}_{\beta}(G)$ and $\mathcal{W} \mathcal{H}_{\beta}(G)$. We use these two random walks to compare the numbers of feasible bases associated with Hamiltonian extreme points in these two polytopes, and explore the efficiency of the wedge constraints. While computational experiments confirm our analytical results on the feasible bases of the polytope $\mathcal{H}_{\beta}(G)$, they reveal that the wedge constraints improve algorithmic efficiency for values of the discount factor $\beta$ sufficiently close to 1 . All these theoretical and computational results support a new conjecture on the feasible bases of the polytope $\mathcal{W} \mathcal{H}_{\beta}(G)$ stated at the end of the paper. 
The remainder of this paper is organized as follows: In Section 2, we review some preliminary results and introduce the polytope $\mathcal{H}_{\beta}(G)$. In Section 3, we characterize the feasible bases of $\mathcal{H}_{\beta}(G)$. In Section 4 , we investigate the expected prevalence of feasible bases of $\mathcal{H}_{\beta}\left(G_{n, p}\right)$ for an input binomial random graph. More precisely, we find the expected number of each type of feasible basis in the random polytope $\mathcal{H}_{\beta}\left(G_{n, p}\right)$. In Section 5, we discuss the results of $[13,12]$ concerning $\mathcal{W} \mathcal{H}_{\beta}(G)$. In Section 6, we investigate the efficiency of this polytope by developing and running two different random walk algorithms on feasible bases of the polytope $\mathcal{W H}_{\beta}(G)$. In Section 7, we conclude with a conjecture on the feasible bases of the polytope $\mathcal{W H}_{\beta}\left(\bar{G}_{n, p}\right)$, where $\bar{G}_{n, p}$ is a Hamiltonian binomial random graph.

\section{Formulation of HCP through discounted MDPs.}

Consider a directed graph $G=(V, E)$, where $V=\{1,2, \ldots, n\}$ is the set of nodes and $E$ is the set of arcs. Throughout this paper, $G$ refers to such a directed graph on $n$ nodes, unless otherwise stated. For each node $i \in V$, we define the in-neighborhood set, $\mathcal{N}^{-}(i)$, and the out-neighborhood set, $\mathcal{N}^{+}(i)$, of $i$ by

$$
\mathcal{N}^{-}(i)=\{j \in V:(j, i) \in E\}, \quad \mathcal{N}^{+}(i)=\{j \in V:(i, j) \in E\}
$$

For a node $i \in V$, its in-degree, denoted by $\operatorname{deg}^{\text {in }}(i)$, is the cardinality of $\mathcal{N}^{-}(i)$, and its out-degree, denoted by $\operatorname{deg}^{\text {out }}(i)$, is the cardinality of $\mathcal{N}^{+}(i)$. The degree of $i \in V$, denoted by $\operatorname{deg}(i)$ is the total number of arcs incident with $i$, that is, $\operatorname{deg}(i)=\operatorname{deg}^{\text {in }}(i)+\operatorname{deg}^{\text {out }}(i)$.

Let us consider a discounted Markov decision process with state space $\{1,2, \ldots, n\}$, action sets $\mathcal{A}(i)$, for each state $i$, and a discount factor $\beta \in(0,1)$. The embedding of the original graph $G$ in such an MDP is based on a one-to-one correspondence of nodes of $G$ with the states of MDP and actions in state $i$ with arcs emanating from node $i$. That is, $\mathcal{A}(i)=\mathcal{N}^{+}(i)$ for $i=1,2, \ldots, n$. The Markovian transition probabilities normally accompanying an MDP are particularly simple in this embedding. Namely, choice of an action corresponding to arc $(i, j)$ results in the transition from state $i$ to state $j$, with probability one.

Using the above embedding, Feinberg [14] converted HCP to a constrained discounted MDP and showed that finding a Hamiltonian cycle is equivalent to finding a structured extreme point of a certain polytope that we shall call the Feinberg polytope $\mathcal{F}_{\beta}(G)$. This result is made precise in Theorem 1, below.

Theorem 1 (Feinberg [14]). Consider the embedding of the graph $G=(V, E)$ in a constrained discounted $M D P$ with a discount factor $\beta$ and the polytope $\mathcal{F}_{\beta}(G)$ characterized by

$$
\begin{array}{rlrl}
\sum_{j \in \mathcal{N}^{+}(1)} y_{1 j}-\beta \sum_{j \in \mathcal{N}^{-}(1)} y_{j 1} & =1, & \\
\sum_{j \in \mathcal{N}^{+}(i)} y_{i j}-\beta \sum_{j \in \mathcal{N}^{-}(i)} y_{j i} & =0 & \\
\sum_{j \in \mathcal{N}^{+}(1)} y_{1 j} & =\frac{1}{1-\beta^{n}}, & \text { for all } i \in V \backslash\{1\} \\
y_{i j} & \geqslant 0 & & \\
& \text { for all }(i, j) \in E
\end{array}
$$

The graph $G$ is Hamiltonian if and only if there exists an extreme point of $\mathcal{F}_{\beta}(G)$ that has exactly $n$ positive coordinates tracing out a Hamiltonian cycle in $G$.

In MDP literature the polyhedral domain defined by constraints (1), (2) and (4) is called the space of discounted occupational measures. These spaces have been studied extensively (see, for example, [2, 19, 22]). Indeed, Feinberg [14] exploited MDP properties of these measures to prove Theorem 1. 
Eshragh et al. [13] transformed the polytope $\mathcal{F}_{\beta}(G)$ by changing variables $x_{i j}:=\left(1-\beta^{n}\right) y_{i j}$ for all $(i, j) \in E$ to produce the polytope $\mathcal{H}_{\beta}(G) \subseteq \mathbb{R}^{|E|}$ defined by the constraints

$$
\begin{array}{rlrl}
\sum_{j \in \mathcal{N}^{+}(1)} x_{1 j}-\beta \sum_{j \in \mathcal{N}^{-}(1)} x_{j 1} & =1-\beta^{n}, & \\
\sum_{j \in \mathcal{N}^{+}(i)} x_{i j}-\beta \sum_{j \in \mathcal{N}^{-}(i)} x_{j i} & =0 & & \text { for all } i \in V \backslash\{1\}, \\
\sum_{j \in \mathcal{N}^{+}(1)} x_{1 j} & =1, & \\
x_{i j} & \geqslant 0 & & \text { for all }(i, j) \in E .
\end{array}
$$

Since values of $\beta$ close to one were shown to be important in [12]-[13], this transformation eliminates numerical instability in (3). In the remainder of this paper, $A$ and $\boldsymbol{b}$ denote the constraint matrix and the right-hand side vector of constraints (5)-(7), respectively. Indeed, $A$ and $\boldsymbol{b}$ depend on the parameter $\beta$. However, for simplicity and because we do not consider more than one value of $\beta$ at a time, we do not make this dependence on $\beta$ explicit. The following definition is motivated directly from Theorem 1 .

Definition 1. Let $\boldsymbol{x}$ be an extreme point of the polytope $\mathcal{H}_{\beta}(G)$. If the positive coordinates of $\boldsymbol{x}$ trace out a Hamiltonian cycle in the graph $G, \boldsymbol{x}$ is called a Hamiltonian extreme point. Otherwise, it is called a non-Hamiltonian extreme point.

As an example, let us construct the polytope $\mathcal{H}_{\beta}(G)$ for the graph given in Figure 1(a):

$$
\begin{aligned}
x_{12}+x_{14}+x_{15}-\beta\left(x_{21}+x_{41}+x_{51}\right) & =1-\beta^{5} \\
x_{21}+x_{23}-\beta\left(x_{12}+x_{32}\right) & =0 \\
x_{32}+x_{34}-\beta\left(x_{23}+x_{43}\right) & =0 \\
x_{41}+x_{43}+x_{45}-\beta\left(x_{14}+x_{34}+x_{54}\right) & =0 \\
x_{51}+x_{54}-\beta\left(x_{15}+x_{45}\right) & =0 \\
x_{12}+x_{14}+x_{15} & =1 \\
x_{12}, x_{14}, x_{15}, x_{21}, x_{23}, x_{32}, x_{34}, x_{41}, x_{43}, x_{45}, x_{51}, x_{54} & \geqslant 0 .
\end{aligned}
$$

It can be written in the form $A \boldsymbol{x}=\boldsymbol{b}, \boldsymbol{x} \geqslant \mathbf{0}$ as follows:

$$
\left.\begin{array}{cccccccccccc}
1 & 1 & 1 & -\beta & 0 & 0 & 0 & -\beta & 0 & 0 & -\beta & 0 \\
-\beta & 0 & 0 & 1 & 1 & -\beta & 0 & 0 & 0 & 0 & 0 & 0 \\
0 & 0 & 0 & 0 & -\beta & 1 & 1 & 0 & -\beta & 0 & 0 & 0 \\
0 & -\beta & 0 & 0 & 0 & 0 & -\beta & 1 & 1 & 1 & 0 & -\beta \\
0 & 0 & -\beta & 0 & 0 & 0 & 0 & 0 & 0 & -\beta & 1 & 1 \\
1 & 1 & 1 & 0 & 0 & 0 & 0 & 0 & 0 & 0 & 0 & 0
\end{array}\right)\left(\begin{array}{l}
x_{12} \\
x_{14} \\
x_{15} \\
x_{21} \\
x_{23} \\
x_{32} \\
x_{34} \\
x_{41} \\
x_{43} \\
x_{45} \\
x_{51} \\
x_{54}
\end{array}\right)=\left(\begin{array}{c}
1-\beta^{5} \\
0 \\
0 \\
0 \\
0 \\
1
\end{array}\right)
$$

It is easy to see that

$$
\left\{\begin{array}{l}
x_{12}=1, x_{23}=\beta, x_{34}=\beta^{2}, x_{45}=\beta^{3}, x_{51}=\beta^{4} \\
x_{14}=x_{15}=x_{21}=x_{32}=x_{41}=x_{43}=x_{54}=0
\end{array}\right.
$$

is an extreme point of $\mathcal{H}_{\beta}(G)$. Furthermore, the set of $\operatorname{arcs}\{(1,2),(2,3),(3,4),(4,5),(5,1)\}$, corresponding to the non-zero variables, traces out a Hamiltonian cycle in the graph $G$. 
Theorem 1 shows that the polytope $\mathcal{H}_{\beta}(G)$ reflects some properties of the Hamiltonian cycles in $G$ and, thus, can be utilized as the basis for a procedure to search for Hamiltonian cycles. This procedure is outlined in Algorithm 1.

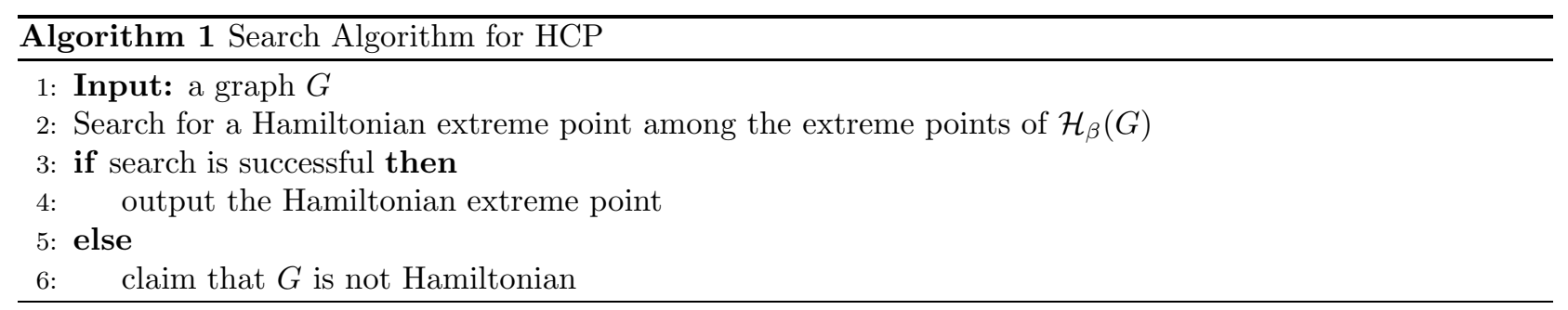

We note that this is only a framework of an algorithm. In order to derive a practical algorithm, it still needs to be specified how the search in Line 2 of Algorithm 1 is implemented. Depending on this implementation, the resulting algorithm can have quite different properties. In particular, if we search by sampling extreme points, we obtain a randomized search algorithm which, with a certain probability, will claim that the input graph $G$ is non-Hamiltonian, although in fact it contains a Hamiltonian cycle. Thus, one may try to alleviate this error probability by designing a clever random search algorithm. The motivation of our work is to better understand the properties of random walk based sampling methods in this context. Since each extreme point can be identified with the set of its corresponding feasible bases. Hence, instead of sampling the extreme points of the polytope $\mathcal{H}_{\beta}(G)$ we can sample its feasible bases. This yields an efficient algorithm provided two technical conditions are satisfied which can be informally stated as follows.

(1) There are sufficiently many extreme points (or feasible bases) corresponding to Hamiltonian cycles, and

(2) The random walk used of sampling converges to the uniform distribution quickly enough.

The first condition ensures that the error probability is small. More precisely, we can bound the probability that among $t$ uniform samples there is none that corresponds to a Hamiltonian cycle. The second condition allows us to bound the number of random walk steps to obtain an approximately uniform sample (see [18] for more details and precise statements).

In order to establish the two conditions listed above for a random walk on the feasible bases of $\mathcal{H}_{\beta}(G)$, it is crucial to understand the structure of these feasible bases. The study of the structure of the extreme points of $\mathcal{H}_{\beta}(G)$ has been initiated in $[10,12]$. However, these references did not consider the structure of the feasible bases. In Section 3, we characterize feasible bases of the polytope $\mathcal{H}_{\beta}(G)$, and describe their structural and geometric properties.

\section{Feasible bases of the polytope $\mathcal{H}_{\beta}(G)$.}

In this section, we consider feasible bases of the polytope $\mathcal{H}_{\beta}(G)$. At the outset, we recall some basic notation and definitions of polytopes, in general.

Definition 2. Let $\mathcal{P} \subseteq \mathbb{R}^{\eta}$ be a polytope defined by the constraints $M \boldsymbol{x}=\boldsymbol{v}, \boldsymbol{x} \geqslant \mathbf{0}$, where $M$ is a $\kappa \times \eta$ matrix of rank $\kappa \leqslant \eta$, and $\boldsymbol{v}$ is an $\eta \times 1$ column vector. An extreme point (or vertex) of $\mathcal{P}$ is a point $\boldsymbol{x} \in \mathcal{P}$ with the property that $M$ has a nonsingular $\kappa \times \kappa$-submatrix $M_{B}$ such that the components of $\boldsymbol{x}$ corresponding to columns not in $M_{B}$ are zero, and those components corresponding to columns in $M_{B}$ are given by $M_{B}^{-1} \boldsymbol{v}$. For a given submatrix $M_{B}$ and the corresponding extreme point $\boldsymbol{x}$, those components of $\boldsymbol{x}$ associated with the columns of $M_{B}$ are called basic variables. The set of all $\kappa$ basic variables is called a feasible basis. Two distinct feasible bases are adjacent if and only if they have exactly $\kappa-1$ common basic variables. An extreme point is called degenerate if it has less than $\kappa$ non-zero components. Otherwise, that is, if it has exactly $\kappa$ non-zero components, it is non-degenerate.

Remark 1. If all extreme points are non-degenerate, then there exists a one-to-one correspondence between feasible bases and extreme points. Otherwise, some extreme points may be associated with more than one feasible basis. Indeed, such degeneracy is generic in applications. 


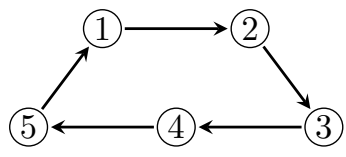

(a) Hamiltonian extreme point

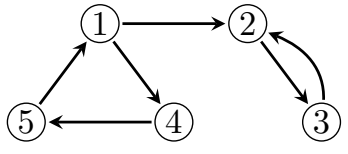

(b) Non-Hamiltonian extreme point

Figure 2. Supports of a Hamiltonian and a non-Hamiltonian extreme point for the graph in Figure 1(a).

The polytope $\mathcal{H}_{\beta}(G)$, where $\beta \in(0,1)$, is generated by $n+1$ linearly independent constraints. Accordingly, each feasible basis of $\mathcal{H}_{\beta}(G)$ has $n+1$ basic variables. As the columns of the constraint matrix $A$ can be identified with the arcs of $G$, characterizing the feasible bases of $\mathcal{H}_{\beta}(G)$ is equivalent to characterizing the arc sets $B \subseteq E$ with $|B|=n+1$ satisfying the following two conditions:

B1: The columns of the $(n+1) \times(n+1)$ matrix $A_{B}$ are linearly independent, where $A_{B}$ is constructed by choosing those columns of $A$ corresponding to $B$.

B2: The inequality $\left(A_{B}\right)^{-1} \boldsymbol{b} \geqslant \mathbf{0}$ is satisfied, which is equivalent to (8).

Henceforth, we use condition $\mathbf{B} \mathbf{1}$ and 'the set $B$ is linearly independent', interchangeably.

We recall some results pertaining to the structure of extreme points of $\mathcal{H}_{\beta}(G)$. For this, we present some definitions.

Definition 3. Let $\boldsymbol{x}$ be an extreme point of $\mathcal{H}_{\beta}(G)$. The support of $\boldsymbol{x}$ is defined to be the set of its nonzero coordinates. Since the variables $x_{i j}$ correspond to arcs of $G$, the support of $\boldsymbol{x}$ can be identified with a subgraph of $G$, which we denote by $\mathcal{S}(G, \boldsymbol{x})$. More precisely, $\mathcal{S}(G, \boldsymbol{x})$ is the graph with node set $V$ and arc set $\left\{(i, j) \in E: x_{i j}>0\right\}$. Since there is no danger of ambiguity, we use the term support of $\boldsymbol{x}$ to refer to the graph $\mathcal{S}(G, \boldsymbol{x})$, the arc set of this graph, or the set of variables $x_{i j}$ corresponding to these arcs.

According to Definition 1, an extreme point $\boldsymbol{x}$ is a Hamiltonian extreme point if and only if the support $\mathcal{S}(G, \boldsymbol{x})$ is a Hamiltonian cycle in $G$. Furthermore, Ejov et al. [10] showed that, for a non-Hamiltonian extreme point $\boldsymbol{x}, \mathcal{S}(G, \boldsymbol{x})$ has a specific structure. In order to state their result, which is summarized in Theorem 2 , we need to define some important paths and cycles in $G$.

Definition 4 (Ejov et al. [10]). A cycle of the form $1 \rightarrow v_{1} \rightarrow \cdots \rightarrow v_{k} \rightarrow 1$ with distinct nodes $v_{i}(i=1, \ldots, k)$ and $k<n-1$ is called a short cycle. A path of the form $1 \rightarrow v_{1} \rightarrow v_{2} \rightarrow \cdots \rightarrow v_{k} \rightarrow v_{j}$ with distinct nodes $v_{i}(i=1, \ldots, k)$ and $1 \leqslant j<k<n$ is called a noose path. The cycle $v_{j} \rightarrow v_{j+1} \rightarrow \cdots \rightarrow v_{k} \rightarrow v_{j}$ is the associated noose cycle.

Example 1. For $n=5$, the $\operatorname{arcs}(1,2),(2,3)$ and $(3,1)$ form a short cycle, and the arcs $(1,2),(2,3),(3,4)$ and $(4,2)$ form a noose path with the associated noose cycle $\{(2,3),(3,4),(4,2)\}$.

Theorem 2 (Ejov et al. [10]). If $\boldsymbol{x}$ is a non-Hamiltonian extreme point of the polytope $\mathcal{H}_{\beta}(G)$, then the support $\mathcal{S}(G, \boldsymbol{x})$ is the union of a short cycle and a noose path. In particular, this implies that the support of a non-Hamiltonian extreme point has a unique node of out-degree two, called the "splitting node", and all other nodes have out-degrees at most one.

Figure 2 illustrates the supports for a Hamiltonian and non-Hamiltonian extreme point of the polytope $\mathcal{H}_{\beta}(G)$, where $G$ is the graph shown in Figure 1(a). More precisely, while the support shown in Figure 2(a) corresponds to a Hamiltonian extreme point with positive components $x_{12}=1, x_{23}=\beta, x_{34}=\beta^{2}, x_{45}=\beta^{3}$ and $x_{51}=\beta^{4}$, the support in Figure 2(b) is associated with a non-Hamiltonian extreme point with positive components $x_{12}=1-\beta^{2}, x_{14}=\beta^{2}, x_{23}=\beta, x_{32}=\beta^{2}, x_{45}=\beta^{3}$ and $x_{51}=\beta^{4}$. In the latter graph, the arc sets $\{(1,4),(4,5),(5,1)\},\{(1,2),(2,3),(3,2)\}$ and $\{(2,3),(3,2)\}$ form the short cycle, noose path and noose cycle, respectively. Furthermore, node 1 is the splitting node for this extreme point.

Motivated by Theorem 2, Eshragh and Filar [12] partitioned the set of non-Hamiltonian extreme points of the polytope $\mathcal{H}_{\beta}(G)$ into four types, based on their supports. More precisely, for a given non-Hamiltonian extreme point, if in the corresponding support: 


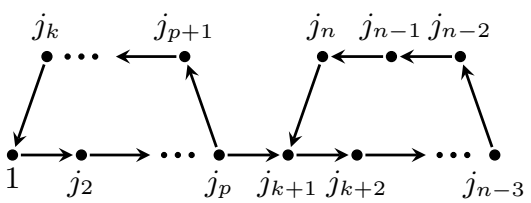

(a) Type 1

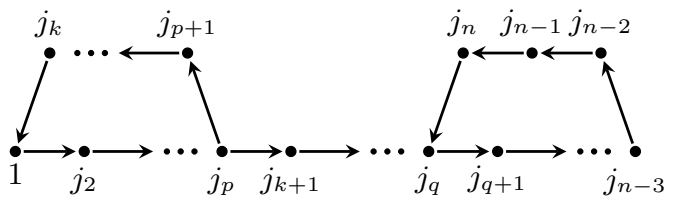

(b) Type 2

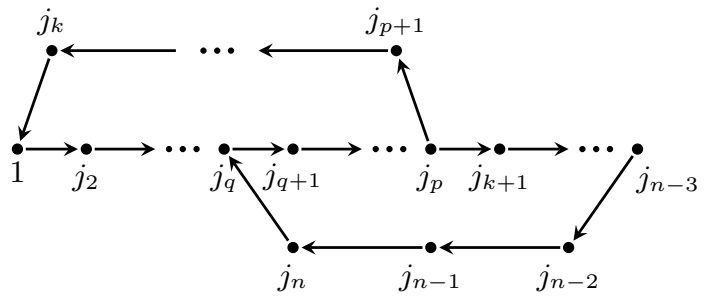

(c) Type 3

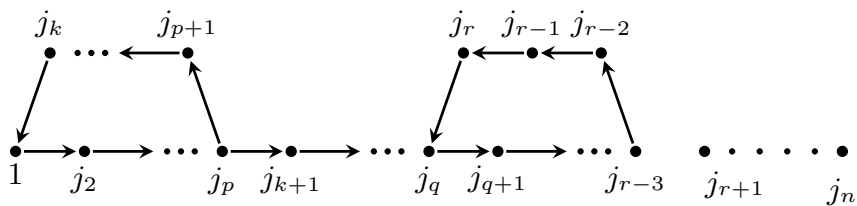

(d) Type 4

Figure 3. Different types of non-Hamiltonian extreme points of the polytope $\mathcal{H}_{\beta}(G)$

Type 1: every node has in-degree at least one, the short cycle and the noose cycle are node-disjoint, and there is one arc connecting the splitting node (which lies on the short cycle) to a node on the noose cycle (Figure 3(a)).

Type 2: every node has in-degree at least one, the short cycle and the noose cycle are node-disjoint, and they are linked by a path of length at least two, connecting the splitting node with a node on the noose cycle (Figure 3(b)).

Type 3: every node has in-degree at least one, and the short cycle and noose cycle have at least one node in common (Figure 3(c)).

Type 4: at least one node has degree zero (Figure 3(d)).

Remark 2. It follows from Theorem 2 that the support of each non-Hamiltonian extreme point of Type 4 can be represented as a support of any of types 1-3 on less then $n$ nodes or a short cycle on less than $n$ nodes with an extra $\operatorname{arc}(i, j)$, where nodes $i$ and $j$ are on the short cycle and node $i$ comes after node $j$.

Definition 5. Let $\boldsymbol{x}$ be an extreme point of the polytope $\mathcal{H}_{\beta}(G)$ with corresponding support $\mathcal{S}(G, \boldsymbol{x})$. A feasible basis that contains the arc set of $\mathcal{S}(G, \boldsymbol{x})$ is called a Hamiltonian basis if $\boldsymbol{x}$ is a Hamiltonian extreme point, and otherwise it is called a non-Hamiltonian basis.

Remark 3. Analogously, the set of feasible bases of the polytope $\mathcal{H}_{\beta}(G)$ can be partitioned into five types: Hamiltonian bases (namely, feasible bases of Type 0) and non-Hamiltonian bases of types 1-4, where a nonHamiltonian basis is Type $i$ for $i=1,2,3,4$ if its corresponding extreme point is Type $i$.

Since the supports of extreme points of types $1-3$ have exactly $n+1$ elements, they are all non-degenerate extreme points. This implies that each extreme point of these types has exactly one corresponding feasible basis forming its support. However, as Hamiltonian and non-Hamiltonian Type 4 extreme points have, respectively, exactly and at most $n$ positive components, they are all degenerate extreme points and accordingly, they may possess several corresponding feasible bases. Thus, the support $\mathcal{S}(G, \boldsymbol{x})$ associated with a Hamiltonian or non-Hamiltonian Type 4 extreme point $\boldsymbol{x}$ does not reveal complete information about the feasible bases corresponding to $\boldsymbol{x}$. For example, Figure 4(a) shows $\mathcal{S}\left(K_{7}, \boldsymbol{x}\right)$, where $K_{7}$ is the complete graph on seven nodes and $\boldsymbol{x}$ is the non-Hamiltonian extreme point of Type 4 with positive coordinates $x_{12}=1, x_{23}=\beta^{3}\left(1+\beta^{2}\right)+\beta$, $x_{32}=\beta^{2}\left(1+\beta^{2}\right), x_{31}=\beta^{6}$. Clearly, in order to construct a feasible basis corresponding to this extreme point, we should add four more appropriate arcs (not necessarily any four arbitrary arcs) to the support given in Figure 4(a). If we try the four arcs $(4,5),(6,5),(7,6)$ and $(7,4)$, as in Figure 4(b), this fails as it would induce 
(7)

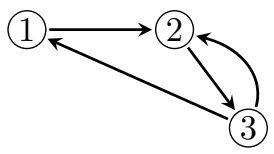

(6)

(5)

(a)

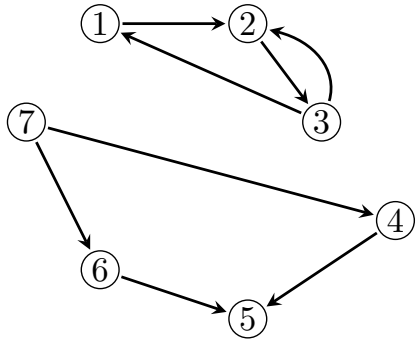

(b)

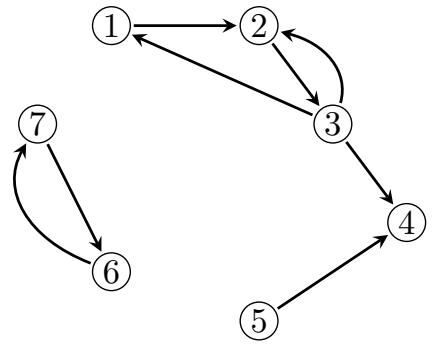

(c)

Figure 4. (a) Support of a non-Hamiltonian extreme point of Type 4 of $\mathcal{H}_{\beta}\left(K_{7}\right)$, and two possible ways to add four arcs leading to a (b) linearly dependent set, and (c) a feasible basis.

linear dependency. However, if we complete the basis with $\operatorname{arcs}(3,4),(5,4),(6,7)$ and $(7,6)$, as in Figure $4(c)$, this results in a feasible basis of Type 4 .

Thus, an important question raised here is which arcs can be added to the support of a degenerate extreme point of the polytope $\mathcal{H}_{\beta}(G)$ to construct a corresponding feasible basis? This question is addressed in Proposition 1 and Theorem 3 for Hamiltonian and non-Hamiltonian Type 4 extreme points, respectively.

Proposition 1. A set $B \subseteq E$ of size $|B|=n+1$ is a Hamiltonian basis of the polytope $\mathcal{H}_{\beta}(G)$ if and only if $B$ contains a Hamiltonian cycle.

Proof. If $B$ is a Hamiltonian basis, it directly follows from Definitions 1 and 5 that it contains a Hamiltonian cycle. So, we just need to show that for any Hamiltonian cycle in $G$ with the arc set $C \subseteq E$ and any arc $(i, j) \in E \backslash C$, the set $B=C \cup\{(i, j)\}$ is a feasible basis for the polytope $\mathcal{H}_{\beta}(G)$. Without loss of generality, assume that

$$
C=\{(1,2),(2,3), \ldots,(n-1, n),(n, 1)\}
$$

and fix any such $B$. In order to show that $B$ is a feasible basis, we need to show that conditions $\mathbf{B} 1$ and $\mathbf{B 2}$ hold. Let $A_{B}$ denote the $(n+1) \times(n+1)$-submatrix of $A$ corresponding to $B$. If $A_{B}$ is invertible and $\boldsymbol{x}$ is the vector whose coordinates corresponding to $B$ are given by $A_{B}^{-1} \boldsymbol{b}$ while all other coordinates are zero, then it has been proved in [13, Lemma 3.2] that $x_{k k+1}=\beta^{k-1}>0$ for $k \in\{1,2, \ldots, n-1\}, x_{n 1}=\beta^{n-1}>0$, and $x_{k l}=0$ for all $(k, l) \in E \backslash C$. So the non-negativity condition $\mathbf{B 2}$ is satisfied whenever the independence condition B1 holds. Hence, we only need to prove that condition B1 holds, that is the set $B$ is linearly independent.

We prove this by contradiction. Let us assume that there exists a non-zero $(n+1) \times 1$ vector $\boldsymbol{x}$ satisfying $A_{B} \boldsymbol{x}=\mathbf{0}$. In this proof, all indices 0 and $n+1$ that appear in arc-indicating subscripts are equivalent to $n$ and 1, respectively; for example, $x_{n n+1}=x_{n 1}$ and $x_{01}=x_{n 1}$. Following constraints (5)-(7), replacing the right-hand sides of (5) and (7) by 0 , this set of linear equations is represented as follows:

$$
\begin{aligned}
x_{k k+1}-\beta x_{k-1} & =0 \\
x_{i i+1}+x_{i j}-\beta x_{i-1} & =0, \\
x_{j j+1}-\beta\left(x_{j-1 j}+x_{i j}\right) & =0, \\
x_{12}+\delta_{i 1} x_{i j} & =0,
\end{aligned}
$$

where $\delta_{i 1}$ is the Kronecker delta, which is equal to one if $i=1$, otherwise zero. If $x_{i j}=0$ then Equation (12) implies that $x_{12}=0$, and then $x_{k+1}=0$ for all $k \in\{1,2, \ldots, n\}$ by induction on $k$, using (9), (10) and (11). Hence, our assumption $\boldsymbol{x} \neq \mathbf{0}$ implies that $x_{i j} \neq 0$. Therefore, without loss of generality, we assume $x_{i j}=1$. As illustrated in Figure 5, there are four possible cases for the arc $(i, j)$ discussed below. We show that in all four cases the non-zero assumption on $\boldsymbol{x}$ is violated, implying that the set $B$ is linearly independent.

Case 1: $i=1,3 \leqslant j \leqslant n$. From Equation (12) we obtain $x_{12}=-1$, and then by Equation (9) and induction on $k$ we have $x_{k k+1}=-\beta^{k-1}$ for $k=1, \ldots, j-1$. So, Equation (11) simplifies to

$$
x_{j+1}-\beta\left(-\beta^{j-2}+1\right)=0 \text {, }
$$




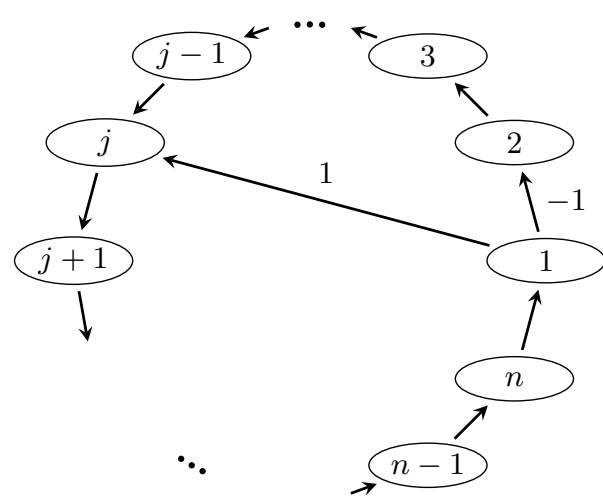

(a) Case 1

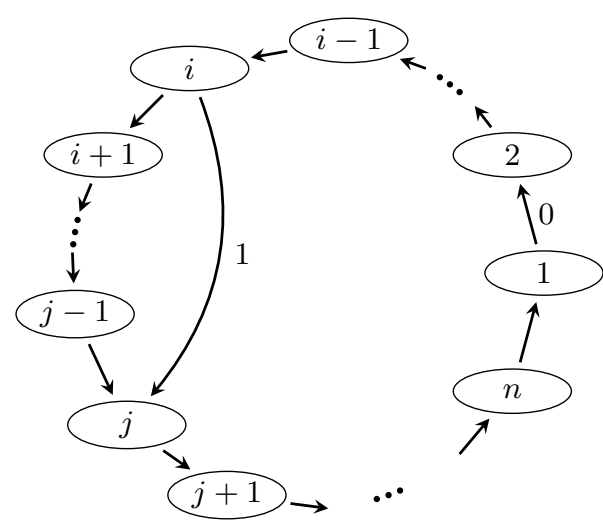

(c) Case 3

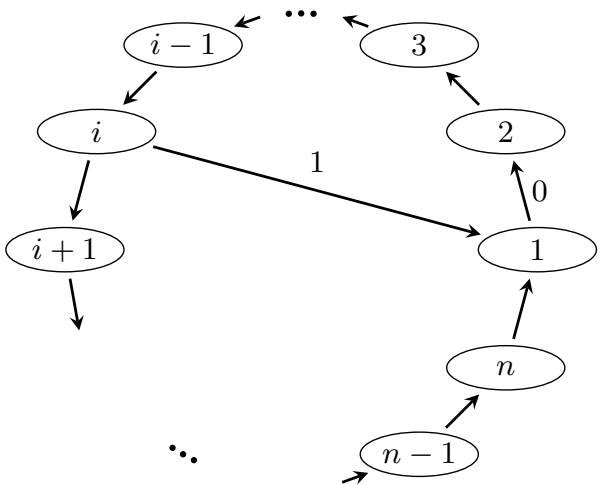

(b) Case 2

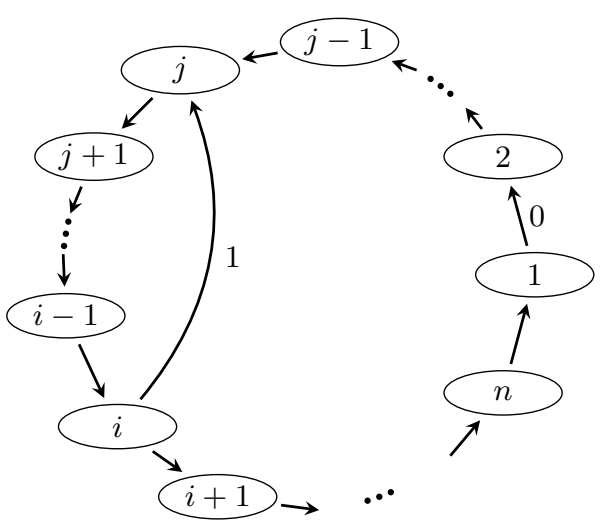

(d) Case 4

Figure 5. Illustration for the four cases discussed in the proof of Proposition 1.

implying that $x_{j+1}=\beta-\beta^{j-1}$. Substituting this value into Equation (9), and proceeding by induction on $k$, we have

$$
x_{k k+1}=\beta^{k-j+1}-\beta^{k-1} \text { for } k=j, j+1, \ldots, n .
$$

Hence, the left-hand side of Equation (10) is

$$
x_{12}+x_{1 j}-\beta x_{n 1}=-1+1-\beta\left(\beta^{n-j+1}-\beta^{n-1}\right)<0 .
$$

In particular, $A_{B} \boldsymbol{x} \neq \mathbf{0}$, which is the required contradiction.

Case 2: $j=1,2 \leqslant i \leqslant n-1$. From Equation (12), we obtain $x_{12}=0$. As in Case 1, we have

$$
x_{k k+1}= \begin{cases}0 & \text { for } k=1,2, \ldots, i-1, \\ -\beta^{k-i} & \text { for } k=i, i+1, \ldots, n .\end{cases}
$$

Hence, the left-hand side of Equation (11) is

$$
x_{12}-\beta\left(x_{n 1}+x_{i 1}\right)=0-\beta\left(-\beta^{n-i}+1\right)<0 .
$$

In particular, $A_{B} \boldsymbol{x} \neq \mathbf{0}$, which is the required contradiction.

Case 3: $2 \leqslant i<j-1 \leqslant n-1$. As in Case 2, we have

$$
x_{k k+1}= \begin{cases}0 & \text { for } k=1,2, \ldots, i-1, \\ -\beta^{k-i} & \text { for } k=i, i+1, \ldots, j-1, \\ \beta^{k-j+1}-\beta^{k-i} & \text { for } k=j, j+1, \ldots, n .\end{cases}
$$




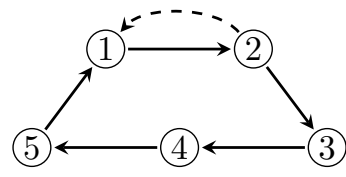

(a)

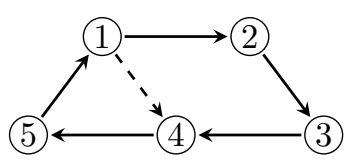

(b)

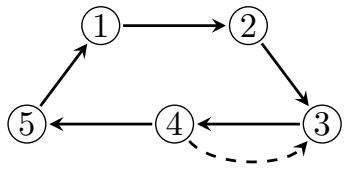

(c)

Figure 6. Three Hamiltonian bases for the graph in Figure 1(a)

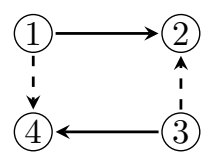

(a) A balanced oriented cycle

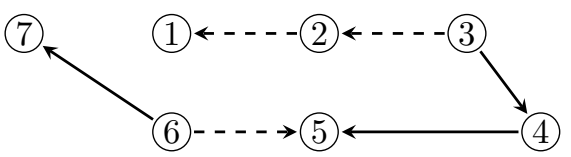

(b) A balanced oriented path

FiguRE 7. Balanced oriented paths

Hence, the left-hand side of Equation (9) for $k=1$ is

$$
x_{12}-\beta x_{n 1}=0-\beta\left(\beta^{n-j+1}-\beta^{n-i}\right)<0 .
$$

In particular, $A_{B} \boldsymbol{x} \neq \mathbf{0}$, which is the required contradiction.

Case 4: $2 \leqslant j<i \leqslant n$. As in Case 2, we have

$$
x_{k k+1}= \begin{cases}0 & \text { for } k=1,2, \ldots, j-1, \\ \beta^{k-j+1} & \text { for } k=j, j+1, \ldots, i-1, \\ \beta^{k-j+1}-\beta^{k-i} & \text { for } k=i, i+1, \ldots, n .\end{cases}
$$

Hence, the left-hand side of Equation (9) for $k=1$ is

$$
x_{12}-\beta x_{n 1}=0-\beta\left(\beta^{n-j+1}-\beta^{n-i}\right)>0 .
$$

In particular, $A_{B} \boldsymbol{x} \neq \mathbf{0}$, which is the required contradiction.

Corollary 1. Every Hamiltonian extreme point of $\mathcal{H}_{\beta}(G)$ corresponds to $|E|-n$ Hamiltonian bases, and any two of these bases are connected by a single variable exchange.

Proof. Proposition 1 implies that a Hamiltonian basis of $\mathcal{H}_{\beta}(G)$ is obtained by adding an arbitrary arc to the arc set of any Hamiltonian cycle in $G$.

For example, Figure 6 illustrates three different Hamiltonian bases of the polytope $\mathcal{H}_{\beta}(G)$ for the input graph $G$ given in Figure 1(a). All those three Hamiltonian bases correspond to the Hamiltonian extreme point with the support displayed in Figure 2(a).

In the remainder of this section, we characterize the non-Hamiltonian bases of the polytope $\mathcal{H}_{\beta}(G)$. In order to formulate the necessary and sufficient conditions on an arc set $B \subseteq E$ to be a non-Hamiltonian basis, we need to introduce the following concept, and develop some preliminary results presented in lemmas 1-4.

Definition 6. Let $P=\left\{\left\{v_{0}, v_{1}\right\},\left\{v_{1}, v_{2}\right\}, \ldots,\left\{v_{k-1}, v_{k}\right\}\right\}$, where $v_{i} \in V$ for $i=0, \ldots, k$, be an undirected path (or cycle if $v_{k}=v_{0}$ ) in the graph $G$. An oriented path (or cycle) of $P$ is denoted by $\vec{P}$ and defined by

$$
\vec{P}=\left\{\left(u_{1}, w_{1}\right),\left(u_{2}, w_{2}\right), \ldots,\left(u_{k}, w_{k}\right)\right\}
$$

where $\left(u_{i}, w_{i}\right) \in\left\{\left(v_{i-1}, v_{i}\right),\left(v_{i}, v_{i-1}\right)\right\}$ for $i=1, \ldots, k$. We call the arc $\left(u_{i}, w_{i}\right)$ a forward arc if $\left(u_{i}, w_{i}\right)=$ $\left(v_{i-1}, v_{i}\right)$ and a backward arc if $\left(u_{i}, w_{i}\right)=\left(v_{i}, v_{i-1}\right)$. For the special cases where $\vec{P}$ is a path of length one or a cycle of length two, that is $\vec{P}=\left\{\left(u_{1}, w_{1}\right)\right\}$ or $\left\{\left(u_{1}, w_{1}\right),\left(w_{1}, u_{1}\right)\right\}$, we use the convention that all arcs are forward arcs. The defect of an oriented path (or cycle) $\vec{P}$ is denoted by $\Delta(\vec{P})$ and defined to be the number of forward arcs minus the number of backward arcs in $\vec{P}$. We say that $\vec{P}$ is balanced if its defect is zero. 
Figures 7(a) and 7(b) depict a balanced oriented cycle and path, respectively. While the solid arcs indicate forward arcs, the dashed arcs are backward arcs.

Remark 4. Any balanced oriented path (or cycle) contains an even number of arcs, where half of them are forward arcs and half are backward arcs. Obviously, a Hamiltonian cycle, short cycle, noose path and noose cycle are non-balanced and all their arcs are forward arcs.

Lemma 1. Let $\boldsymbol{x}=\left(x_{i j}\right)_{|E| \times 1}$, where $(i, j) \in E$, be a real-valued vector whose support is an oriented cycle $\vec{C} \subseteq E$ that does not contain node 1 . Then $A \boldsymbol{x}=\mathbf{0}$ if and only if the following three conditions are satisfied:

(i) $x_{i j}=-x_{i k}$ if node $i$ has out-degree 2 in $\vec{C}$ and $(i, j),(i, k) \in \vec{C}$;

(ii) $x_{j i}=-x_{k i}$ if node $i$ has in-degree 2 in $\vec{C}$ and $(j, i),(k, i) \in \vec{C}$;

(iii) $x_{i j}=\beta x_{k i}$ if node $i$ has in-and out-degree 1 in $\vec{C}$ and $(i, j),(k, i) \in \vec{C}$.

Proof. This is an immediate consequence of constraint (6).

Lemma 2. Let $\vec{C} \subseteq E$ be an oriented cycle not containing node 1 . The set of columns of $A$ corresponding to the arcs in $\vec{C}$ is linearly dependent if and only if $\vec{C}$ is balanced.

Proof. We first prove the 'only if' statement by showing that if the set of columns of $A$ corresponding to $\vec{C}$ is linearly dependent, then $\vec{C}$ is a balanced oriented cycle. As all results in this proof are derived in 'if and only if' condition, the converse, that is the 'if' statement, can easily be shown by following the 'only if' proof backward. Let $A_{\vec{C}}$ denote the $(n+1) \times k$ submatrix constructed by choosing the columns of $A$ corresponding to arcs in the oriented cycle $\vec{C}=\left\{e_{1}, e_{2}, \ldots, e_{k}\right\}$, where $e_{l}=\left(i_{l}, j_{l}\right) \in E$ for $l=1, \ldots, k$ and $k<n$. Define $\vec{P}_{l}=\left\{e_{1}, e_{2}, \ldots, e_{l}\right\}$ as the oriented subpath of the oriented cycle $\vec{C}$ for $l=1, \ldots, k$. Let $i^{*}$ be the common node of $\operatorname{arcs} e_{1}$ and $e_{k}$, and $\sigma_{l}$ be the number of nodes in $\vec{P}_{l}$ having in- or out-degree 2. For $l=k$, we do not consider $i^{*}$ in the calculation of $\sigma_{k}$. From our linear dependency assumption, we know that there exists a non-zero $k \times 1$ vector $\boldsymbol{x}$ such that $A_{\vec{C}} \boldsymbol{x}=\mathbf{0}$. Lemma 1 prescribes that $x_{i_{l} j_{l}} \neq 0$ for all $\left(i_{l}, j_{l}\right) \in \vec{C}$. So, without loss of generality, we can re-scale $\boldsymbol{x}$ and define

$$
x_{i_{1} j_{1}}= \begin{cases}1 & \text { if } i_{1}=i^{*} \text { (i.e., } e_{1} \text { is a forward arc), } \\ \beta^{-1} & \text { if } j_{1}=i^{*} \text { (i.e., } e_{1} \text { is a backward arc). }\end{cases}
$$

By applying Lemma 1 and induction on $l$, we can show the following equality for the component of vector $\boldsymbol{x}$ corresponding to the last arc in $\vec{P}_{l}$, for $l=1, \ldots, k$ :

$$
x_{i_{l} j_{l}}= \begin{cases}(-1)^{\sigma_{l}} \beta^{\Delta\left(\vec{P}_{l}\right)-1} & \text { if } e_{l}=\left(i_{l}, j_{l}\right) \text { is a forward arc, } \\ (-1)^{\sigma_{l}} \beta^{\Delta\left(\vec{P}_{l}\right)} & \text { if } e_{l}=\left(i_{l}, j_{l}\right) \text { is a backward arc. }\end{cases}
$$

As the number of nodes with in- or out-degree 2 in the oriented cycle $\vec{C}$ is even, $i^{*}$ has in- or out-degree 2 , if and only if $\sigma_{k}$ is odd. For node $i^{*}$ in $\vec{P}_{k}$ (which is identical to $\vec{C}$ ), we have the following four possible cases:

Case 1: $i_{1}=i_{k}=i^{*}$. In this case, $e_{1}=\left(i^{*}, j_{1}\right)$ is a forward arc and $e_{k}=\left(i^{*}, j_{k}\right)$ is a backward arc. As the out-degree of node $i^{*}$ is $2, \sigma_{k}$ is an odd number. Consequently, from equations (13) and (14) we have $x_{i^{*} j_{1}}=1$ and $x_{i^{*} j_{k}}=-\beta^{\Delta(\vec{C})}$, respectively. However, Lemma 1 prescribes that $x_{i^{*} j_{1}}=-x_{i^{*} j_{k}}$. Hence, in this case, we must have $\Delta(\vec{C})=0$.

Case 2: $i_{1}=j_{k}=i^{*}$. In this case, both $e_{1}=\left(i^{*}, j_{1}\right)$ and $e_{k}=\left(i_{k}, i^{*}\right)$ are forward arcs. As the in- and out-degree of node $i^{*}$ are $1, \sigma_{k}$ is an even number. Consequently, from equations (13) and (14) we have $x_{i^{*} j_{1}}=1$ and $x_{i_{k} i^{*}}=\beta^{\Delta(\vec{C})-1}$, respectively. However, Lemma 1 prescribes that $x_{i^{*} j_{1}}=\beta x_{i_{k} i^{*}}$. Hence, in this case, we must have $\Delta(\vec{C})=0$.

Case 3: $j_{1}=i_{k}=i^{*}$. In this case, both $e_{1}=\left(i_{1}, i^{*}\right)$ and $e_{k}=\left(i^{*}, j_{k}\right)$ are backward arcs. As the inand out-degree of node $i^{*}$ is $1, \sigma_{k}$ is an even number. Consequently, from equations (13) and (14) we have $x_{i_{1} i^{*}}=\beta^{-1}$ and $x_{i^{*} j_{k}}=\beta^{\Delta(\vec{C})}$, respectively. However, Lemma 1 prescribes that $x_{i^{*} j_{k}}=\beta x_{i_{1} i^{*}}$. Hence, in this case, we must have $\Delta(\vec{C})=0$. 
Case 4: $j_{1}=j_{k}=i^{*}$. In this case, $e_{1}=\left(i_{1}, i^{*}\right)$ is a backward arc and $e_{k}=\left(i_{k}, i^{*}\right)$ is a forward arc. As the in-degree of node $i^{*}$ is $2, \sigma_{k}$ is an odd number. Consequently, from equations (13) and (14) we have $x_{i_{1} i^{*}}=\beta^{-1}$ and $x_{i_{k} i^{*}}=-\beta^{\Delta(\vec{C})-1}$, respectively. However, Lemma 1 prescribes that $x_{i_{1} i^{*}}=-x_{i_{k} i^{*}}$. Hence, in this case, we must have $\Delta(\vec{C})=0$.

Thus, $\vec{C}$ is a balanced oriented cycle.

Lemma 3. If $H=(W, B)$, where $W \subseteq V \backslash\{1\}$ and $B \subseteq E$, is a connected subgraph of $G$ with $|B|=|W|$, then the set of columns of $A$ corresponding to arcs in $B$ is linearly dependent if and only if the unique oriented cycle in $B$ is balanced.

Proof. If the oriented cycle in $B$ is balanced then $B$ is linearly dependent by Lemma 2. For the converse, suppose that $B$ is linearly dependent and let $B^{\prime} \subseteq B$ be a minimal dependent subset. This implies that there exists a nonzero vector $\boldsymbol{x}$ with $A_{B^{\prime}} \boldsymbol{x}=\mathbf{0}$, where $A_{B^{\prime}}$ is a submatrix constructed by the columns of $A$ corresponding to $B^{\prime}$, such that for every $\operatorname{arc}(i, j) \in B^{\prime}$, the component $x_{i j} \neq 0$. If the subgraph $H^{\prime}=\left(W, B^{\prime}\right)$ had a node $i$ of degree 1 , then constraint (6) for this node would imply that $x_{i j}=0$ (if $\left.(i, j) \in B^{\prime}\right)$ or $x_{j i}=0$ (if $(j, i) \in B^{\prime}$ ). Since this contradicts the minimality of $B^{\prime}$, we conclude that $B^{\prime}$ is exactly the arc set of the oriented cycle in $B$. Lemma 2 prescribes that $B^{\prime}$ is balanced.

In Lemma 4 and Theorem 3 we use the notation $H=(V, B)$, where $B \subseteq E$ with $|B|=n+1$, is a subgraph of the graph $G$ with connected components $H_{1}, H_{2}, \ldots, H_{m}$. Moreover, $H_{k}=\left(V_{k}, B_{k}\right)$, where $V_{k}$ and $B_{k}$ denote the set of nodes and arcs comprising the connected component $H_{k}$ for $k=1, \ldots, m$, respectively. Without loss of generality, we assume that $1 \in V_{1}$. Let $\rho\left(H_{1}\right)$ denote a subgraph of $H_{1}$ constructed by repeatedly removing all nodes in $H_{1}$ with degree equal to 1 . We use the notation $V_{\rho}$ and $B_{\rho}$ to denote the node set and arc set of the graph $\rho\left(H_{1}\right)$. It should be noted that the concept of constructing the subgraph $\rho\left(H_{1}\right)$ is analogous to the 2-core of the graph $H_{1}$ (see, for example [5] or [21]).

Lemma 4. Consider the subgraph $H=(V, B)$ with connected components $H_{1}, \ldots, H_{m}$. Let $A_{B}$ be a submatrix of $A$ corresponding to the arcs of $B$. If $A_{B}$ is an invertible matrix and $\boldsymbol{x}=A_{B}^{-1} \boldsymbol{b}$, then

(i) $\left|B_{k}\right|= \begin{cases}\left|V_{1}\right|+1 & \text { for } k=1, \\ \left|V_{k}\right| & \text { for } k=2, \ldots, m ;\end{cases}$

(ii) $x_{i j}=0$ for each $(i, j) \in B \backslash B_{\rho}$.

Proof. (i) Since $A_{B}$ is an invertible $(|V|+1) \times|B|$ square matrix, $\operatorname{rank}\left(A_{B}\right)=|V|+1=|B|=n+1$. Moreover, it follows from constraints (5)-(7) that $A_{B}$ has a block structure, with non-overlapping blocks $A_{B_{1}}, \ldots, A_{B_{m}}$ such that $A_{B_{1}}$ is a $\left(\left|V_{1}\right|+1\right) \times\left|B_{1}\right|$ matrix corresponding to the nodes and arcs in $V_{1}$ and $B_{1}$, respectively, and $A_{B_{k}}$ is a $\left|V_{k}\right| \times\left|B_{k}\right|$ matrix corresponding to the nodes and arcs in $V_{k}$ and $B_{k}$, respectively, for $k=2, \ldots, m$. Since there are no arcs between distinct components $H_{i}$ and $H_{j}$ for $i, j \in\{1, \ldots, m\}$ and $i \neq j$, and $\operatorname{rank}\left(A_{B}\right)=|V|+1$, we should have $\operatorname{rank}\left(A_{B_{1}}\right)=\left|V_{1}\right|+1$ and $\operatorname{rank}\left(A_{B_{k}}\right)=\left|V_{k}\right|$, for $k=2, \ldots, m$. Similarly, as $\operatorname{rank}\left(A_{B}\right)|B|$, we should also have $\operatorname{rank}\left(A_{B_{k}}\right)=A_{B_{k}}$ equal to $\left|B_{k}\right|$, for $k=1, \ldots, m$. Thus,

$$
\left|B_{k}\right|= \begin{cases}\left|V_{1}\right|+1 & \text { for } k=1, \\ \left|V_{k}\right| & \text { for } k=2, \ldots, m .\end{cases}
$$

(ii) Let $B^{\prime} \subseteq B$ be the support of $\boldsymbol{x}$. We define $\boldsymbol{x}^{k}$ as the restriction of $\boldsymbol{x}$ to the coordinates in $B_{k}$ for $k=2, \ldots, m$. Constraint (6) implies that $A_{B_{k}} \boldsymbol{x}^{k}=\mathbf{0}$, and since $A_{B_{k}}$ has full column rank, we obtain $\boldsymbol{x}^{k}=\mathbf{0}$ for $k=2, \ldots, m$. Hence, $B^{\prime} \subseteq B_{1}$. Moreover, if the subgraph $H^{\prime}=\left(V_{1}, B^{\prime}\right)$ has a node $i$ of degree 1 , then constraint (6) for node $i$ implies that $x_{i j}=0$ (if $(i, j) \in B^{\prime}$ ) or $x_{j i}=0$ (if $(j, i) \in B^{\prime}$ ), which contradicts the definition of $B^{\prime}$. Thus, all nodes in the subgraph $H^{\prime}$ have degree at least 2 implying that $B^{\prime} \subseteq B_{\rho}$.

Theorem 3. Consider a subgraph $H=(V, B)$, with connected components $H_{1}, \ldots, H_{m}, m \in\{1, \ldots,\lfloor(n-$ $1) / 2\rfloor\}$. The arc set $B$ is a non-Hamiltonian basis of the polytope $\mathcal{H}_{\beta}(G)$ if and only if the following three conditions are satisfied: 


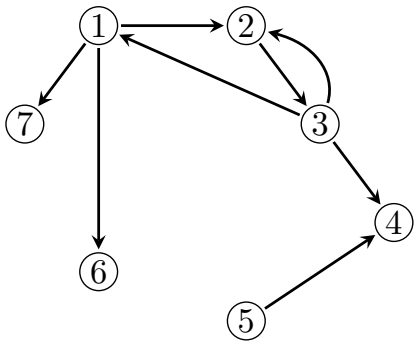

(a)

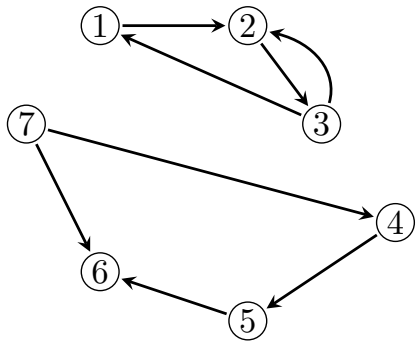

(b)

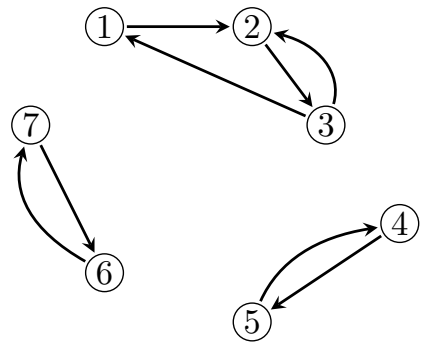

(c)

Figure 8. Three non-Hamiltonian bases of Type 4 corresponding to the support in Figure 4(a)

(i) $\left|B_{k}\right|= \begin{cases}\left|V_{1}\right|+1 & \text { for } k=1 \\ \left|V_{k}\right| & \text { for } k=2, \ldots, m ;\end{cases}$

(ii) If $m>1, H_{k}$ does not contain a balanced oriented cycle, for $k=2, \ldots, m$;

(iii) The subgraph $\rho\left(H_{1}\right)$ is the union of a short cycle and a noose path.

Proof. Let $B$ be a non-Hamiltonian basis of the polytope $\mathcal{H}_{\beta}(G)$ corresponding to an extreme point $\boldsymbol{x}$, and let $A_{B}$ be the associated submatrix of $A$. Since $B$ is a basis, $A_{B}$ is invertible. Consequently, conditions (i) and (ii) follow from lemmas 4 and 3, respectively. Now, consider the subgraph $\rho\left(H_{1}\right)$. By Lemma 4 , we know that the support $\mathcal{S}(G, \boldsymbol{x})$ is a subgraph of $\rho\left(H_{1}\right)$. Moreover, as we remove an equal number of nodes and arcs to construct $\rho\left(H_{1}\right)$, condition (i) implies that $\left|B_{\rho}\right|=\left|V_{\rho}\right|+1$. It follows that the support $\mathcal{S}(G, \boldsymbol{x})$ is identical to the graph $\rho\left(H_{1}\right)$. Hence, by Theorem 2, we can conclude condition (iii), that is $\rho\left(H_{1}\right)$ is the union of a short cycle and a noose path.

Conversely, suppose that conditions (i)-(iii) are satisfied. In order to prove that $B$ is a non-Hamiltonian basis, we should only show that conditions B1 and B2 hold, implying that $B$ is a feasible basis. Then, condition (iii) with Theorem 2 prescribe that $B$ is a non-Hamiltonian basis. If $A_{B_{k}}$ denotes a submatrix of $A$ corresponding to the node and arc sets $V_{k}$ and $B_{k}$, respectively, for $k=1, \ldots, m$, condition (i) implies that each $A_{B_{k}}$ is a square matrix and so $A_{B}$ has a block diagonal structure. Hence, we have $\operatorname{det}\left(A_{B}\right)=\Pi_{k=1}^{m} \operatorname{det}\left(A_{B_{k}}\right)$ and $A_{B}$ is invertible if $A_{B_{k}}$ is invertible for all $k=1, \ldots, m$. Consequently, to show that $A_{B}$ is invertible, we need to show that each submatrix $A_{B_{k}}$ is invertible. Lemma 3 with conditions (i)-(ii) imply that $A_{B_{k}}$ is invertible for $k=2, \ldots, m$. It remains to show that $A_{B_{1}}$ is invertible as well. Let $\boldsymbol{\lambda} \in \mathbb{R}^{\left|B_{1}\right|}$ be a vector satisfying $A_{B_{1}} \boldsymbol{\lambda}=\mathbf{0}$. As in the proof of Lemma 3, we show that $\lambda_{i j}=0$ for all $(i, j) \in B_{1} \backslash B_{\rho}$. From Theorem 2 and condition (iii), the columns of $A_{B_{1}}$ corresponding to arcs in $B_{\rho}$ are linearly independent; hence $\lambda_{i j}=0$ for for all $(i, j) \in B_{\rho}$. Consequently, we have $\boldsymbol{\lambda}=\mathbf{0}$, implying that the columns of $A_{B_{1}}$ are linearly independent. So, condition B1 holds. Finally, Theorem 2 and condition (iii) confirm that condition B2 holds as well.

Remark 5. Theorem 3 implies that the arc set of a spanning subgraph $H$ of $G$ is a non-Hamiltonian basis if and only if (i) $H$ has exactly $n+1$ arcs, (ii) every node in $H$ has positive degree, (iii) $H$ does not contain a balanced oriented cycle, and (iv) $H$ contains a short cycle and a noose path.

Remark 6. Theorem 3 describes the structure of all non-Hamiltonian bases of types $1-4$. However, as the supports of non-degenerate non-Hamiltonian extreme points of types 1-3 have exactly $n+1$ arcs, for their corresponding feasible bases, we have $H=H_{1}$ and the result of Theorem 3 will be consistent with Theorem 2 . So, the main contribution of Theorem 3 can be regarded for the structure of degenerate non-Hamiltonian bases of Type 4, which is utilized in Section 4 to derive their expected prevalence in the polytope $\mathcal{H}_{\beta}(G)$ when the input graph $G$ is random.

As Figure 4 (b) contains a balanced oriented cycle $\vec{C}=\{(4,5),(6,5),(7,6),(7,4)\}$, it is now readily seen that it is not associated with a feasible basis of the polytope $\mathcal{H}_{\beta}\left(K_{7}\right)$. However, as Figure 4(c) satisfies all conditions provided in Theorem 3, it corresponds to a non-Hamiltonian basis of Type 4. Figure 8 shows three more non-Hamiltonian bases corresponding to the support displayed in Figure 4(a). 


\section{EXPeCted numbers of FEASible Bases of $\mathcal{H}_{\beta}$-POLytope For RANDOM GRAPhS.}

As discussed in Section 2, the correspondence between the sets of Hamiltonian cycles in the graph $G$ and feasible bases of the polytope $\mathcal{H}_{\beta}(G)$ can be exploited to develop an algorithm that searches for Hamiltonian cycles. One key issue influencing the efficiency of such an algorithm is the existence of a sufficiently large number of Hamiltonian bases. More precisely, we require the ratio of the number of Hamiltonian bases to nonHamiltonian bases to be bounded below by $1 / h(n)$, where $h(n)$ is a polynomial in $n$. Then for a Hamiltonian input graph $G$ the probability of not finding a Hamiltonian basis in the first $\alpha h(n)$ iterations would be expected to decay rapidly with $\alpha$ if the search algorithm sampled feasible bases uniformly at random. For instance, if Algorithm 1 were modified to search on feasible bases (instead of extreme points), according to a uniform distribution, then we could expect to find a Hamiltonian cycle, with very high probability, in polynomial time. Thus it is important to investigate the prevalence of Hamiltonian bases in $\mathcal{H}_{\beta}(G)$.

In this section, we exploit the structural results from Section 3 to derive the expected prevalence of each of the five types of feasible bases of the polytope $\mathcal{H}_{\beta}(G)$ where $G$ is a binomial random graph and show that the majority of these are non-Hamiltonian bases of Type 4. Thus, the expected number of Hamiltonian bases of this polytope is exponentially small, when compared to the expected number of non-Hamiltonian bases of Type 4. Fortunately, in [12], it was shown that these undesirable feasible bases can be eliminated by the addition of $2 n-2$ lower and upper bound constraints.

The following definition is needed for the ensuing discussion. For an introduction to the theory of general random graphs, we refer the interested reader to Bollobás [6].

Definition 7. For a positive integer $n$ and a fixed probability $p \in[0,1]$, the directed binomial random graph on $n$ nodes, denoted by $G_{n, p}$, is a random directed graph on $n$ nodes where each ordered pair of distinct nodes is, independently, connected by an arc with probability $p$.

As discussed in Section 3, the feasible bases of the polytope $\mathcal{H}_{\beta}(G)$ can be identified by certain subgraphs of $G$ on $n$ nodes and $n+1$ arcs. For $k=0,1,2,3,4$, let $f_{k}(n)$ denote the number of subgraphs of the complete graph $K_{n}$ corresponding to the feasible bases of Type $k$ of the polytope $\mathcal{H}_{\beta}\left(K_{n}\right)$. Recall that the feasible bases of Type 0 are referred as Hamiltonian bases. Label all $f_{k}(n)$ feasible bases of Type $k$ of the polytope $\mathcal{H}_{\beta}\left(K_{n}\right)$, $1, \ldots, f_{k}(n)$. Define the binary random variable $I_{i}(k)$ that takes the value of one, if the $i^{t h}$ feasible basis of Type $k$ of the polytope $\mathcal{H}_{\beta}\left(K_{n}\right)$ appears in the random polytope $\mathcal{H}_{\beta}\left(G_{n, p}\right)$, and otherwise zero. Clearly,

$$
\mathbf{E}\left(I_{i}(k)\right)=p^{n+1},
$$

where $\mathbf{E}$ denotes the expected value. Although $I_{i}(k)$ are dependent random variables, as the expectation is a linear operator, we have

$$
\mathbf{E}\left(\sum_{i=1}^{f_{k}(n)} I_{i}(k)\right)=\sum_{i=1}^{f_{k}(n)} \mathbf{E}\left(I_{i}(k)\right)=f_{k}(n) p^{n+1} .
$$

Equation (15) implies that the expected number of feasible bases of Type $k$ of the polytope $\mathcal{H}_{\beta}\left(G_{n, p}\right)$ equals $f_{k}(n) p^{n+1}$ for $k=0, \ldots, 4$. In particular, the ratio between the expected number of Hamiltonian bases and the expected total number of feasible bases in the random polytope $\mathcal{H}_{\beta}\left(G_{n, p}\right)$ is independent of $p$ and equals $f_{0}(n) /\left[f_{0}(n)+\cdots+f_{4}(n)\right]$. Theorem 4 provides the expected number of feasible bases of types $0-3$ as well as a lower bound on the expected number of feasible bases of Type 4 by determining the value of $f_{k}(n)$ for $k=0, \ldots, 3$ and finding a lower bound on $f_{4}(n)$.

Theorem 4. Consider the binomial random graph $G_{n, p}$ and the corresponding polytope $\mathcal{H}_{\beta}\left(G_{n, p}\right)$. The expected number of

(i) Hamiltonian bases is $(n-2) n ! p^{n+1}$;

(ii) non-Hamiltonian bases of Type 1 is $\frac{1}{2}(n-3) n ! p^{n+1}$;

(iii) non-Hamiltonian bases of Type 2 is $\frac{1}{6}(n-4)(n-3)(n+1)(n-1) ! p^{n+1}$; 
(iv) non-Hamiltonian bases of Type 3 is $\frac{1}{6}(n-2)(n-1) n ! p^{n+1}$;

(v) non-Hamiltonian bases of Type 4 is at least $(n-1)(n-2)(n-3)^{n-5} 2^{n-4} p^{n+1}$.

Proof.

(i) The complete graph $K_{n}$ possesses $n(n-1)$ arcs and $(n-1)$ ! Hamiltonian cycles. By Proposition 1 and Corollary 1, for each Hamiltonian cycle, any of the $n(n-2)$ remaining arcs can be added to form a feasible basis. It follows that $f_{0}(n)=(n-2) n$ !.

(ii) A non-Hamiltonian basis of Type 1 consists of a short cycle of length $k \in\{2,3, \ldots, n-2\}$, a noose cycle of length $n-k$, and an arc joining them. For a fixed $k$, there are

- $\left(\begin{array}{c}n-1 \\ k-1\end{array}\right)(k-1)$ ! ways to construct a short cycle;

- $(n-k-1)$ ! ways to construct a noose cycle from the remaining nodes, after fixing the short cycle;

- $\left(\begin{array}{c}k \\ 1\end{array}\right)\left(\begin{array}{c}n-k \\ 1\end{array}\right)$ options to choose a node from the short cycle and the noose cycle to connect them together. Consequently,

$$
f_{1}(n)=\sum_{k=2}^{n-2} \frac{(n-1) !}{(n-k) !}(n-k-1) ! k(n-k)=(n-1) ! \sum_{k=2}^{n-2} k=\frac{1}{2}(n-3) n ! .
$$

(iii) A non-Hamiltonian basis of Type 2 consists of a short cycle of length $k \in\{2,3, \ldots, n-3\}$, a noose cycle of length $l \in\{2,3, \ldots, n-k-1\}$, and a path of length $n-(k+l)+1$ joining them. For fixed $k$ and $l$, there are

- $\left(\begin{array}{c}n-1 \\ k-1\end{array}\right)(k-1)$ ! ways to construct a short cycle;

- $\left(\begin{array}{c}n-k \\ l\end{array}\right)(l-1)$ ! ways to construct a noose cycle, after fixing the short cycle;

- $(n-k-l)$ ! ways to permute the remaining nodes to construct the connecting path from the short cycle to the noose cycle;

- $\left(\begin{array}{l}k \\ 1\end{array}\right)\left(\begin{array}{l}l \\ 1\end{array}\right)$ options to choose a node from the short cycle and a node from the noose cycle to connect them through the path constructed in (iii).

Consequently,

$$
\begin{array}{r}
f_{2}(n)=\sum_{k=2}^{n-3} \sum_{l=2}^{n-k-1} \frac{(n-1) !}{(n-k) !} \cdot \frac{(n-k) !}{l(n-k-l) !}(n-k-l) ! k l=\sum_{k=2}^{n-3} \sum_{l=2}^{n-k-1} k(n-1) !=(n-1) ! \sum_{k=2}^{n-3}(n-k-2) k \\
=(n-1) !\left((n-2) \sum_{k=2}^{n-3} k-\sum_{k=2}^{n-3} k^{2}\right)=\frac{1}{6}(n-4)(n-3)(n+1)(n-1) ! .
\end{array}
$$

(iv) For a non-Hamiltonian basis of Type 3 with a short cycle of length $k \in\{2,3, \ldots, n-1\}$, there are

- $\left(\begin{array}{l}n-1 \\ k-1\end{array}\right)(k-1)$ ! ways to construct a short cycle;

- $(n-k)$ ! ways to permute the remaining nodes, after fixing the short cycle;

- $\left(\begin{array}{c}k-1 \\ 1\end{array}\right)$ options to choose a splitting node on the short cycle;

- $\left(\begin{array}{l}l \\ 1\end{array}\right)$ options to choose a node with in-degree 2 on the short cycle, where $l$ is the location of the splitting node on the short cycle starting from node 1.

Consequently,

$$
f_{3}(n)=\sum_{k=2}^{n-1} \sum_{l=1}^{k-1} \frac{(n-1) !}{(n-k) !}(n-k) ! l=(n-1) ! \sum_{k=2}^{n-1} \frac{k(k-1)}{2}=\frac{1}{6}(n-2)(n-1) n ! .
$$

(v) In order to establish a lower bound for $f_{4}(n)$, following Remark 2, we bound the number of feasible bases of Type 3 corresponding to non-Hamiltonian extreme point $\boldsymbol{x}$ such that their supports have only three nodes including one short cycle and one noose cycle, each of length 2 . If $\boldsymbol{x}$ is non-Hamiltonian extreme point of the polytope $\mathcal{H}_{\beta}\left(K_{n}\right)$ with this structure, there are $\left(\begin{array}{c}n-1 \\ 1\end{array}\right)\left(\begin{array}{c}n-2 \\ 1\end{array}\right)=(n-1)(n-2)$ ways to construct the support $\mathcal{S}\left(K_{n}, \boldsymbol{x}\right)$. Any undirected tree spanning the remaining $n-3$ nodes can be oriented in $2^{n-4}$ ways, and for each of these orientations there is always an additional arc that can be added without creating a balanced oriented cycle. As a consequence of Theorem 3 and Remark 5 , the result is a feasible 
basis of Type 4 corresponding to $\boldsymbol{x}$. By Cayley's formula (see, for instance, [1, Chapter 30]), the number of spanning trees on $n-3$ labeled nodes is equal to $(n-3)^{n-5}$. Thus, we have

$$
f_{4}(n) \geqslant(n-1)(n-2)(n-3)^{n-5} 2^{n-4} .
$$

Corollary 2. In the polytope $\mathcal{H}_{\beta}\left(G_{n, p}\right)$, for sufficiently large $n$, we have

(i)

$$
\frac{\boldsymbol{E}(\text { Number of feasible bases of Type } 4)}{\boldsymbol{E}(\text { Total number of feasible bases })} \geqslant 1-\frac{n^{11 / 2}}{e^{n} 2^{n-9}}
$$

(ii)

$$
\frac{\boldsymbol{E}(\text { Number of Hamiltonian bases })}{\boldsymbol{E}(\text { Total number of feasible bases })} \leqslant \frac{n^{9 / 2}}{e^{n-1} 2^{n-9}} .
$$

Proof.

(i) The quotient on the left-hand side is

$$
\begin{aligned}
& \frac{f_{4}(n)}{f_{0}(n)+f_{1}(n)+f_{2}(n)+f_{3}(n)+f_{4}(n)}=1-\frac{f_{0}(n)+f_{1}(n)+f_{2}(n)+f_{3}(n)}{f_{0}(n)+f_{1}(n)+f_{2}(n)+f_{3}(n)+f_{4}(n)} \\
& \geqslant 1-\frac{f_{0}(n)+f_{1}(n)+f_{2}(n)+f_{3}(n)}{f_{4}(n)}
\end{aligned}
$$

Using Stirling's formula to bound the factorials in parts (i)-(iv) of Theorem 4,

$$
f_{0}(n)+f_{1}(n)+f_{2}(n)+f_{3}(n)=\frac{1}{3}\left(n^{3}-7 n+6\right)(n-1) ! \leqslant \frac{1}{3} n^{2} n ! \leqslant n^{n+5 / 2} e^{-n} .
$$

Furthermore, for sufficiently large $n$, we have

$$
\begin{gathered}
f_{4}(n) \geqslant(n-1)(n-2)(n-3)^{n-5} 2^{n-4}=n^{n-3}\left(\frac{n-1}{n}\right)\left(\frac{n-2}{n}\right)\left(\frac{n-3}{n}\right)^{n-5} 2^{n-4} \\
=n^{n-3} 2^{n-4}\left(1-\frac{1}{n}\right)\left(1-\frac{2}{n}\right)\left(\left(1-\frac{3}{n}\right)^{n / 3}\right)^{3(n-5) / n} \\
\geqslant n^{n-3} 2^{n-4}\left(1-\frac{2}{n}\right)^{2}\left(\frac{1}{e}\right)^{3} \geqslant n^{n-3} 2^{n-4}\left(\frac{27}{32}\right)\left(\frac{1}{3}\right)^{3}=n^{n-3} 2^{n-9} .
\end{gathered}
$$

Thus, we obtain

$$
\frac{f_{4}(n)}{f_{0}(n)+f_{1}(n)+f_{2}(n)+f_{3}(n)+f_{4}(n)} \geqslant 1-\frac{n^{n+5 / 2} e^{-n}}{n^{n-3} 2^{n-9}}=1-\frac{n^{11 / 2}}{e^{n} 2^{n-9}} .
$$

(ii) From part (i) of Theorem 4 and Inequality (16) as well as utilizing Stirling's formula, the left-hand side is bounded above as follows:

$$
\frac{f_{0}(n)}{f_{0}(n)+f_{1}(n)+f_{2}(n)+f_{3}(n)+f_{4}(n)} \leqslant \frac{f_{0}(n)}{f_{4}(n)} \leqslant \frac{(n-2) n !}{n^{n-3} 2^{n-9}} \leqslant \frac{n^{9 / 2}}{e^{n-1} 2^{n-9}} .
$$

Remark 7 . Corollary 2 shows that even the expected number of feasible bases of Type 4 corresponding to a portion of supports on three nodes is exponentially larger than the expected number of feasible bases of types 0, 1, 2 and 3 all together. Thus, non-Hamiltonian bases of Type 4 can make up the majority of feasible bases of the polytope $\mathcal{H}_{\beta}(G)$. This result is supported numerically in Section 6 . 


\section{REDUCING THE FEASIBLE REGION.}

Theorem 4 and Corollary 2 show that the main obstacle to finding a Hamiltonian basis of the polytope $\mathcal{H}_{\beta}(G)$ may lie in the abundance of non-Hamiltonian bases of Type 4. As indicated by Corollary 2 , as the number of nodes of the graph $G, n$, increases, the ratio between the numbers of non-Hamiltonian bases of Type 4 and all feasible bases of the polytope $\mathcal{H}_{\beta}(G)$ could tend to one exponentially fast. It is therefore of interest to modify the polytope $\mathcal{H}_{\beta}(G)$ in such a way that all non-Hamiltonian bases of Type 4 are eliminated, while all Hamiltonian feasible bases are preserved. To this end, Eshragh and Filar [12] added 2( $n-1)$ of the so-called wedge constraints $(17)$, to $\mathcal{H}_{\beta}(G)$, thereby defining a modified polytope $\mathcal{W} \mathcal{H}_{\beta}(G)$ with the following constraints set:

$$
\begin{gathered}
\boldsymbol{x} \in \mathcal{H}_{\beta}(G), \\
\beta^{n-1} \leqslant \sum_{j \in \mathcal{N}^{+}(i)} x_{i j} \leqslant \beta \quad \text { for all } i \in V \backslash\{1\} .
\end{gathered}
$$

In [12] it was shown that, when $\beta$ is sufficiently close to one, the wedge constraints (17) excise what is, typically, the majority of non-Hamiltonian bases. This result is summarized in Theorem 5 .

Theorem 5 (Eshragh and Filar [12]). Consider the graph $G=(V, E)$ on $n$ nodes and the corresponding polytopes $\mathcal{H}_{\beta}(G)$ and $\mathcal{W} \mathcal{H}_{\beta}(G)$. For values of $\beta \in\left(\left(1-\frac{1}{n-2}\right)^{\frac{1}{n-2}}, 1\right)$, the only possible common extreme points of polytopes $\mathcal{H}_{\beta}(G)$ and $\mathcal{W H}_{\beta}(G)$ are the Hamiltonian extreme points and the non-Hamiltonian extreme points of Type 1.

Our results in Section 4 show that, for a given binomial random graph, the majority of non-Hamiltonian bases are of Type 4. Since the result of Theorem 5 can clearly be extended from extreme points to feasible bases, removing non-Hamiltonian extreme points of Type 4 may be interpreted as the efficiency of the wedge constraints. However, the wedge constraints may also add new extreme points. In order to fully investigate the efficiency of the wedge constraints, we need to determine the structure and prevalence of feasible bases of these new extreme points of the polytope $\mathcal{W H}_{\beta}(G)$. Analogous to Eshragh et al. [13], we introduce the concept of quasi-Hamiltonian bases for $\mathcal{W} \mathcal{H}_{\beta}(G)$ as follows.

Definition 8. Let $\boldsymbol{x}=\left(x_{i j}\right) \in \mathbb{R}^{|E|}$ be an extreme point of $\mathcal{W} \mathcal{H}_{\beta}(G)$. The extreme point $\boldsymbol{x}$ is called quasiHamiltonian if any path $i_{1} \rightarrow i_{2} \rightarrow \cdots \rightarrow i_{n} \rightarrow i_{n+1}$, where $i_{k+1} \in \arg \max _{j \in \mathcal{N}^{+}\left(i_{k}\right)}\left\{x_{i_{k} j}\right\}$ for $k=1, \ldots, n$ is a Hamiltonian cycle in $G$. In this case, any feasible basis corresponding to $\boldsymbol{x}$ is called a quasi-Hamiltonian basis.

As an example, consider the following quasi-Hamiltonian extreme point of the polytope $\mathcal{W H}_{\beta}\left(K_{6}\right)$ for $\beta=0.999$ :

$$
\begin{array}{r}
x_{12}=1.00000, x_{26}=0.999000, x_{34}=0.994013 ; x_{36}=0.000997, x_{45}=0.995010, x_{51}=0.995010 \\
x_{54}=0.001993, x_{63}=0.996006, x_{65}=0.00299101,
\end{array}
$$

and all the other $x_{i j}$ are zero. We can easily see that this extreme point corresponds to the Hamiltonian cycle $1 \rightarrow 2 \rightarrow 6 \rightarrow 3 \rightarrow 4 \rightarrow 5 \rightarrow 1$. Any feasible basis of the polytope $\mathcal{W H}_{\beta}\left(K_{6}\right)$ corresponding to this particular extreme point is called a quasi-Hamiltonian basis.

Remark 8. Evidently, every Hamiltonian extreme point in the polytope $\mathcal{H}_{\beta}(G)$ is also a quasi-Hamiltonian extreme point in the polytope $\mathcal{W H}_{\beta}(G)$. Furthermore, quasi-Hamiltonian bases increase the pool of desirable feasible bases.

In the next section, we develop two algorithms to study the effectiveness of the wedge constraints in removing non-Hamiltonian bases and the role of the discount parameter $\beta$. We present promising numerical results regarding the efficiency of the modified polytope $\mathcal{W} \mathcal{H}_{\beta}(G)$ over $\mathcal{H}_{\beta}(G)$, in the sense that the prevalence of quasi-Hamiltonian bases among all feasible bases of $\mathcal{W H}_{\beta}(G)$ appears much greater than the prevalence of Hamiltonian bases among all feasible bases of $\mathcal{H}_{\beta}(G)$. This means that, based on our numerical evidence, the wedge constraints do not add an excessive number of non-Hamiltonian bases. 
TABLE 1. No. of steps required to find one Hamiltonian cycle

\begin{tabular}{cr}
\hline Random Graph & No. of Steps \\
\hline $\bar{G}_{10,3 / 10}$ & 6 \\
$\bar{G}_{20,3 / 20}$ & 454 \\
$\bar{G}_{30,3 / 30}$ & 1358 \\
$\bar{G}_{40,3 / 40}$ & 5714 \\
$\bar{G}_{50,3 / 50}$ & 4392 \\
$\bar{G}_{60,3 / 60}$ & 6230 \\
$\bar{G}_{70,3 / 70}$ & 6387 \\
\hline
\end{tabular}

\section{Computational Results.}

The polytopes $\mathcal{H}_{\beta}(G)$ and $\mathcal{W H}_{\beta}(G)$ introduced in sections 2 and 5 , respectively, can be used as the basis of a search algorithm for HCP (Algorithm 1). As indicated earlier, one key improvement to enhance the efficiency of such an algorithm would be to increase the ratio of Hamiltonian bases to all feasible bases in the polytopes. Corollary 2 indicates that, on average, the vast majority of feasible bases of the polytope $\mathcal{H}_{\beta}\left(G_{n, p}\right)$ are non-Hamiltonian bases of Type 4 and therefore the number of Hamiltonian bases is not sufficiently large for our purposes. Theorem 5 show that the wedge constraints cut off three types of non-Hamiltonian extreme points of $\mathcal{H}_{\beta}(G)$. However, they may also introduce a number of additional extreme points and consequently new feasible bases to the polytope $\mathcal{W H}_{\beta}(G)$. In order to compare the polytopes $\mathcal{H}_{\beta}(G)$ and $\mathcal{W H}_{\beta}(G)$, we develop two random walk algorithms on feasible bases of the two polytopes.

A random walk algorithm to find one Hamiltonian cycle is Algorithm 2. More precisely, this algorithm runs a simple uniform random walk on the feasible bases of the polytope $\mathcal{W H}_{\beta}(G)$ for an input Hamiltonian graph $G$ and stops when it finds a quasi-Hamiltonian basis and reports the total number of steps to reach that basis.

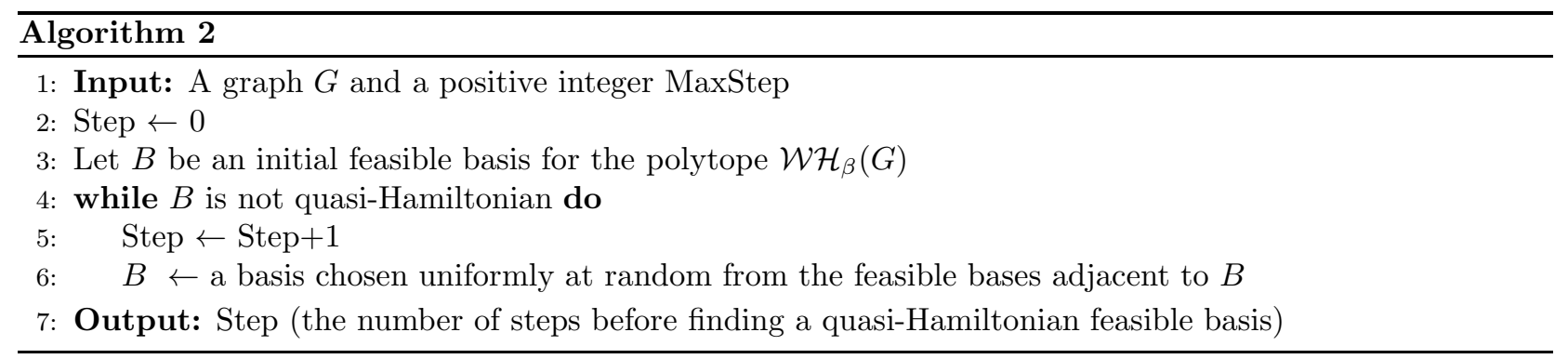

The random walk step in Line 6 of Algorithm 2 is implemented as follows. The bases adjacent to $B$ are given by pairs of a leaving variable and an entering variable chosen from the set of basic variables and non-basic variables, respectively. Each of these pairs is checked for feasibility, and the feasible ones are listed together as the set of feasible bases adjacent to $B$. Finally, one of the adjacent feasible bases is chosen uniformly at random.

We implemented Algorithm 2 in Matlab R2015b and tested it on a range of Hamiltonian binomial random graphs $\bar{G}_{n, p}$. The latter are merely binomial random graphs $G_{n, p}$ augmented by insertion (if necessary) of arcs corresponding to one randomly generated Hamiltonian cycle, thereby ensuring the Hamiltonicity of $\bar{G}_{n, p}$. In Table 1 , we report the performance of Algorithm 2 on several of these graphs with $p=3 / n, n=10, \ldots, 70$ and $\beta=0.999$.

It is important to note the slow growth of the required iterations with increases in $n$. For NP-complete problems, such as HCP, doubling or tripling $n$ would normally dramatically increase the number of iterations. Furthermore, we emphasize the crucial role of the wedge constraint and the parameter $\beta$. 
We modified Algorithm 2 slightly by replacing $\mathcal{W H}_{\beta}(G)$ and quasi-Hamiltonian with $\mathcal{H}_{\beta}(G)$ and Hamiltonian, respectively, and running it on the same $\bar{G}_{n, p}$ graphs reported in Table 1 . In all cases, we failed to find Hamiltonian cycles in 1,000,000 steps. Furthermore, all one million of the non-Hamiltonian basis identified in the process were of Type 4 . This is consistent with Corollary 2 and Remark 7.

Another interesting feature of the polytope $\mathcal{W H}_{\beta}(G)$ is the role played by the parameter $\beta$. Theorem 5 implies that the wedge constraints can be efficient when $\beta$ is sufficiently close to one. In order to test this result numerically, we implemented Algorithm 2 on $\bar{G}_{30,3 / 30}$ for various values of $\beta$. We were interested in determining how increases in the parameter $\beta$ would affect the search for quasi-Hamiltonian bases. The results are given in Table 2. The first column contains the chosen values of $\beta$, while the second column shows the number of steps that the algorithm took to find a quasi-Hamiltonian basis. When Algorithm 2 did not find a quasi-Hamiltonian basis in 30,000 steps, we reported 'fail'. Table 2 demonstrates the crucial role of $\beta$ in the polytope $\mathcal{W H}_{\beta}(G)$ where fewer steps are required to find a quasi-Hamiltonian basis for larger values of $\beta$. These numerical experiments are aligned with theoretical results stated in Theorem 5.

Remark 9. Analogous to the proof of Theorem 4.3 in [12], we can show that if a given graph $G$ is Hamiltonian, then Algorithm 2 almost surely converges to a quasi-Hamiltonian basis in a finite number of steps.

Next we wished to demonstrate, numerically, the prevalence of quasi-Hamiltonian bases in the random polytope $\mathcal{W H}_{\beta}\left(\bar{G}_{n, p}\right)$. Hence, Algorithm 2 has been modified as described in Algorithm 3.

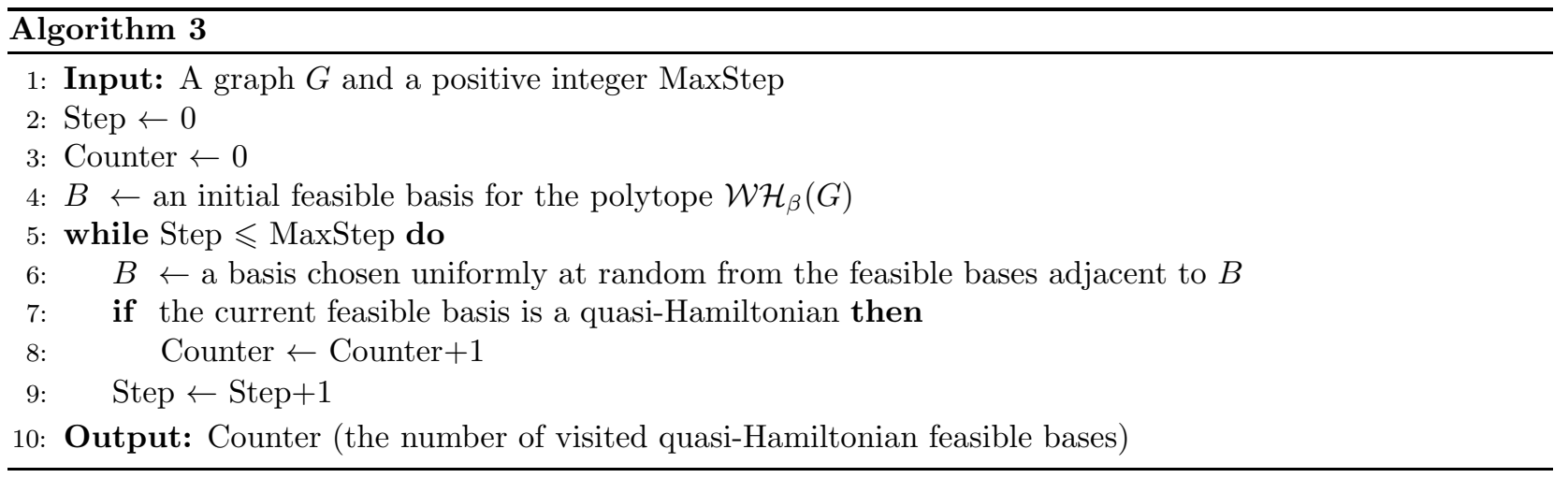

Thus, Algorithm 3 counts the number of quasi-Hamiltonian bases visited during a prescribed number of steps, say MaxStep, for an input graph $G$. We ran this algorithm for 100,000 steps on the same seven $\bar{G}_{n, p}$ graphs as reported above and with $\beta=0.999$. The results are summarized in Table 3 , where the second column lists the number of visited feasible bases (out of 100,000 ) that were quasi-Hamiltonian. These numbers are remarkably high and appear to be a direct consequence of both the nature of wedge constraints and the high value of $\beta$. This is consistent with Theorem 5 and suggests that many of the additional extreme points created by wedge constraints may be quasi-Hamiltonian.

\section{ConClusion}

This paper should be seen as a continuation of results reported in Feinberg [14], Ejov et al. [10], Eshragh et al. [13] and Eshragh and Filar [12]. The original idea of exploiting the discounted occupational measures in MDP in which a graph $G$ is embedded led to algorithmic insights only after the structure of the associated polytope $\mathcal{H}_{\beta}(G)$ had been examined in more detail. In particular, the analysis of extreme points of $\mathcal{H}_{\beta}(G)$ presented in $[10,12]$ necessitated a more precise analysis of their corresponding feasible bases (Proposition 1 and Theorem 3). The subsequent analysis of the expected prevalence of the five types of feasible bases in the random polytope $\mathcal{H}_{\beta}\left(G_{n, p}\right)$ (Theorem 4) exposes the essential difficulty of HCP by demonstrating the asymptotic dominance of non-Hamiltonian bases of Type 4 (Corollary 2). On the positive side, it follows from Theorem 4 that, asymptotically, the expected number of Hamiltonian bases is twice that of non-Hamiltonian bases of Type 1 in the random polytope $\mathcal{H}_{\beta}\left(G_{n, p}\right)$. This is important in view of Theorem 5 (proved in [12]), which suggests that adding the wedge constraints and thereby converting $\mathcal{H}_{\beta}(G)$ to $\mathcal{W H}_{\beta}(G)$ results in a 
TABLE 2. Dependence of Algorithm 2 on $\beta$ for the input graph $\bar{G}_{30,3 / 10}$

\begin{tabular}{cr}
\hline Value of $\beta$ & No. of Steps \\
\hline 0.5 & fail \\
0.6 & fail \\
0.7 & fail \\
0.8 & fail \\
0.9 & fail \\
0.99 & 1944 \\
0.995 & 1139 \\
0.999 & 1001 \\
0.9995 & 84 \\
0.9999 & 23 \\
\hline
\end{tabular}

TABLE 3. Number of quasi-Hamiltonian bases visited by Algorithm 3

\begin{tabular}{cr}
\hline Random Graph & No. of Q-HBs \\
\hline $\bar{G}_{10,3 / 10}$ & 70,197 \\
$\bar{G}_{20,3 / 20}$ & 47,897 \\
$\bar{G}_{30,3 / 30}$ & 6629 \\
$\bar{G}_{40,3 / 40}$ & 34,434 \\
$\bar{G}_{50,3 / 50}$ & 19,472 \\
$\bar{G}_{60,3 / 60}$ & 1790 \\
$\bar{G}_{70,3 / 70}$ & 2863 \\
\hline
\end{tabular}

polytope where searches for quasi-Hamiltonian bases may be more effective. Recall that in Section 2 we noted that the random walk based approach leads to an efficient algorithm provided (i) there are sufficiently many feasible bases corresponding to Hamiltonian cycles, and (ii) the convergence of the random walk to the uniform distribution is sufficiently fast. While Corollary 2 is bad news regarding condition (i) for $\mathcal{H}_{\beta}(G)$, the numerical results of Section 6 indicate that the situation is much better for $\mathcal{W H}_{\beta}(G)$. More precisely, we make the following conjecture on the feasible bases of the random polytope $\mathcal{W} \mathcal{H}_{\beta}\left(\bar{G}_{n, p}\right)$.

Conjecture 1. There exist positive constants $c, \delta$ and $k$, such that for all values $\beta \in\left(1-e^{-c n}, 1\right)$, with high probability, the expected proportion of feasible bases of $\mathcal{W} \mathcal{H}_{\beta}\left(\bar{G}_{n, p}\right)$ that are quasi-Hamiltonian is at least $\delta / n^{k}$.

This conjecture states that there are sufficiently many quasi-Hamiltonian feasible bases in the polytope $\mathcal{W H}_{\beta}(G)$ for large values of $\beta$. This statement was supported by numerical results reported in Section 6 . Proving this conjecture would be a big step towards an efficient variant of Algorithm 1 by sampling feasible bases of $\mathcal{W H}_{\beta}(G)$ instead of extreme points of $\mathcal{H}_{\beta}(G)$.

\section{ACKNOWLEDGMENTS.}

The authors are grateful to Professor Michael Saunders, an anonymous reviewer and the Area Editor for their invaluable comments that helped improve the previous version of this paper. The second author wishes to acknowledge the support from the Australian Research Council under grants DP150100618 and DP160101236.

\section{REFERENCES}

[1] Martin Aigner, Günter M. Ziegler, Karl H. Hofmann, and Paul Erdős. Proofs from THE BOOK. Springer, 2010.

[2] Eitan Altman. Constrained Markov decision processes, volume 7. CRC Press, 1999.

[3] David L. Applegate, Robert E. Bixby, Vasek Chvatal, and William J. Cook. The Traveling Salesman Problem: A Computational Study. Princeton University Press, 2011.

[4] Konstantin Avrachenkov, Ali Eshragh, and Jerzy A. Filar. On transition matrices of Markov chains corresponding to Hamiltonian cycles. Annals of Operations Research, 243(1-2):19-35, 2016.

[5] Bela Bollobás. The evolution of sparse graphs. In Graph Theory and Combinatorics, Proc. Cambridge Combinatorial Conf. in honor of Paul Erdös, pages 35-57. Academic Press, London, 1984.

[6] Béla Bollobás. Modern Graph Theory, chapter Random Graphs, pages 215-252. Springer, 1998.

[7] Vivek S. Borkar and Jerzy A. Filar. Markov chains, Hamiltonian cycles and volumes of convex bodies. Journal of Global Optimization, 55(3):633-639, 2011.

[8] Vladimir Ejov, Jerzy A. Filar, and Jacek Gondzio. An interior point heuristic for the Hamiltonian cycle problem via Markov decision processes. Journal of Global Optimization, 29(3):315-334, 2004. 
[9] Vladimir Ejov, Jerzy A. Filar, Walter Murray, and Giang T. Nguyen. Determinants and longest cycles of graphs. SIAM Journal on Discrete Mathematics, 22(3):1215-1225, 2008.

[10] Vladimir Ejov, Jerzy A. Filar, Michael Haythorpe, and Giang T. Nguyen. Refined MDP-based branchand-fix algorithm for the Hamiltonian cycle problem. Mathematics of Operations Research, 34(3):758-768, 2009.

[11] Vladimir Ejov, Nelly Litvak, Giang T. Nguyen, and Peter G. Taylor. Proof of the Hamiltonicity-Trace conjecture for singularly perturbed Markov chains. Journal of Applied Probability, 48(04):901-910, 2011.

[12] Ali Eshragh and Jerzy A. Filar. Hamiltonian cycles, random walks, and discounted occupational measures. Mathematics of Operations Research, 36(2):258-270, 2011.

[13] Ali Eshragh, Jerzy A. Filar, and Michael Haythorpe. A hybrid simulation-optimization algorithm for the Hamiltonian cycle problem. Annals of Operations Research, 189(1):103-125, 2011.

[14] Eugene A. Feinberg. Constrained discounted Markov decision processes and Hamiltonian cycles. Mathematics of Operations Research, 25(1):130-140, 2000.

[15] Jerzy A. Filar and Dmitry Krass. Hamiltonian cycles and Markov chains. Mathematics of Operations Research, 19(1):223-237, 1994.

[16] Jerzy A. Filar and Asghar Moeini. Hamiltonian cycle curves in the space of discounted occupational measures. Annals of Operations Research, page in press, 2015.

[17] Michael R. Garey, David S. Johnson, and R. Endre Tarjan. The planar Hamiltonian circuit problem is NP-complete. SIAM Journal on Computing, 5(4):704-714, 1976.

[18] Mark Jerrum. Counting, sampling and integrating: algorithms and complexity. Springer Science \& Business Media, 2003.

[19] Lodewijk Cornelis Maria Kallenberg. Linear programming and finite Markovian control problems, volume 148 of Mathematical Centre Tracts. Mathematical Centre, Amsterdam, 1983.

[20] Nelly Litvak and Vladimir Ejov. Markov chains and optimality of the Hamiltonian cycle. Mathematics of Operations Research, 34(1):71-82, 2009.

[21] Tomasz Luczak. Size and connectivity of the $k$-core of a random graph. Discrete Mathematics, 91(1): 61-68, 1991.

[22] Martin L. Puterman. Markov Decision Processes: Discrete Stochastic Dynamic Programming. John Wiley \& Sons, 2014. 